\title{
Single-Cell Electrical Stimulation Using CMOS-Based High-Density Microelectrode Arrays
}

\author{
Silvia Ronchi ${ }^{1 *}$, Michele Fiscella ${ }^{1,2}$, Camilla Marchetti ${ }^{1}$, Vijay Viswam ${ }^{1,2}$, Jan Müller ${ }^{1,2}$, \\ Urs Frey ${ }^{1,2}$ and Andreas Hierlemann ${ }^{1}$ \\ ${ }^{1}$ Department of Biosystems Science and Engineering, ETH Zürich, Basel, Switzerland, ${ }^{2}$ MaxWell Biosystems AG, Basel, \\ Switzerland
}

Non-invasive electrical stimulation can be used to study and control neural activity in the brain or to alleviate somatosensory dysfunctions. One intriguing prospect is to precisely stimulate individual targeted neurons. Here, we investigated single-neuron current and voltage stimulation in vitro using high-density microelectrode arrays featuring 26,400 bidirectional electrodes at a pitch of $17.5 \mu \mathrm{m}$ and an electrode area of $5 \times 9 \mu \mathrm{m}^{2}$. We determined optimal waveforms, amplitudes and durations for both stimulation modes. Owing to the high spatial resolution of our arrays and the close proximity of the

OPEN ACCESS

Edited by:

Udo Kraushaar:

Natural and Medical Sciences

Institute, Germany

Reviewed by:

Paolo Massobrio,

Università di Genova, Italy Dries Braeken,

Interuniversity Microelectronics Centre

(IMEC), Belgium

*Correspondence:

Silvia Ronchi

silvia.ronchi@bsse.ethz.ch

Specialty section: This article was submitted to

Neural Technology, a section of the journal

Frontiers in Neuroscience

Received: 14 December 2018 Accepted: 22 February 2019

Published: 13 March 2019

Citation:

Ronchi S, Fiscella M, Marchetti C, Viswam V, Müller J, Frey $U$ and Hierlemann A (2019) Single-Cell

Electrical Stimulation Using CMOS-Based High-Density

Microelectrode Arrays.

Front. Neurosci. 13:208.

doi: 10.3389/fnins.2019.00208 electrodes to the respective neurons, we were able to stimulate the axon initial segments (AIS) with charges of less than $2 \mathrm{pC}$. This resulted in minimal artifact production and reliable readout of stimulation efficiency directly at the soma of the stimulated cell. Stimulation signals as low as $70 \mathrm{mV}$ or $100 \mathrm{nA}$, with pulse durations as short as $18 \mu \mathrm{s}$, yielded measurable action potential initiation and propagation. We found that the required stimulation signal amplitudes decreased with cell growth and development and that stimulation efficiency did not improve at higher electric fields generated by simultaneous multi-electrode stimulation.

Keywords: HD-MEA, voltage stimulation, current stimulation, single-cell stimulation, axon initial segment

\section{INTRODUCTION}

Electrical stimulation (Rattay, 1999; Merrill, 2010) is a consolidated technique that has been widely used to study neuronal networks (Kumar et al., 2016; Wülfing et al., 2018), to treat brain diseases (Perlmutter and Mink, 2006; Benabid et al., 2009) and somatosensory dysfunctions (Brindley and Lewin, 1968; Shannon, 1983, 1985; Sekirnjak et al., 2008; Tsai et al., 2012; Grosberg et al., 2017; Greenberg et al., 2018; Fan et al., 2019), and to enhance moto-rehabilitation (Raspopovic et al., 2014; Armenta Salas et al., 2018). Electrical stimulation was combined with prosthetic implants in a variety of in vivo applications (Woodson et al., 2009; Dagnelie, 2012). For example, epiretinal implants feature electrodes that deliver an electric signal to neurons located in the retina, in close proximity to the optic nerve. The purpose of eye implants is to artificially substitute nonfunctional retina layers that fail to transduce light into electrical signals for the brain (Brindley and Lewin, 1968; Sekirnjak et al., 2008; Tsai et al., 2012; Grosberg et al., 2017; Fan et al., 2019). Electrical stimulation is employed as well in cochlear implants, where electrodes are used for hearing restoration by stimulating specific cochlear areas depending on sound frequency (Shannon, 1983, 1985; Greenberg et al., 2018). Neural stimulation has been used in the field of prosthesis embodiment for paralyzed patients to restore sensations in upper and lower limbs 
(Raspopovic et al., 2014; Armenta Salas et al., 2018). Furthermore, deep brain electrical stimulation of the subthalamic nucleus is used in Parkinson's treatment (Perlmutter and Mink, 2006; Benabid et al., 2009) to reliably suppress and control the patients' tremor.

Although a large variety of electrical stimulation-based prostheses exists, a major limitation of these devices is their low spatial resolution in delivering stimulation signals and the difficulty to locally constrain the electrical field to attain accurate and precise stimulation of preferably individual single cells. Indeed, blurred images, low sound resolution, inaccurate proprioceptive sensations, and adverse neurocognitive effects may be the results of imprecise electrical stimulation. The described shortcomings motivated us to explore stimulation parameters and regimes and to develop methods for accurate and precise charge injection. In vitro technologies enable to explore a large set of parameters to electrically stimulate neurons in cultures and 3D tissues or slices. Results and findings of in vitro studies of electrical stimulation can potentially be translated and optimized for in vivo applications (e.g., epiretinal implants and cochlear implants).

In vitro high-density microelectrode arrays (HD-MEAs) facilitate electrical-signal readout and stimulation of multiple neurons simultaneously at high-spatiotemporal resolution (Obien et al., 2015). Traditional microelectrode arrays have been used since 1970s (Thomas et al., 1972) for extracellular electrophysiology. Several studies have been carried out to investigate electrical stimulation parameters in neuronal cultures with the aim to find the most efficient way to elicit neuronal responses (Wagenaar et al., 2004; Ahmadian et al., 2011; Grosberg et al., 2017). Although the principles of electrical stimulation have been established, the large electrode size and pitch did not allow to perform stimulation at subcellular resolution and to demonstrate reliable single-neuron targeting.

The introduction of HD-MEAs in complementarymetal-oxide-semiconductor (CMOS) technology for in vitro applications (Eversmann et al., 2003; Berdondini et al., 2009; Frey et al., 2010; Ballini et al., 2014; Bertotti et al., 2014; Viswam et al., 2016; Tsai et al., 2017) enabled to obtain high spatial resolution and a large overall sensing area. Hundreds of researchers worldwide at universities, research institutes and pharmaceutical industries are currently using different CMOSbased HD-MEAs for their studies. CMOS-based HD-MEAs are also commercially available from several suppliers, including Multichannel Systems (Germany), 3Brain (Switzerland) or MaxWell Biosystems (Switzerland). With the advent of neurons derived from human induced pluripotent stem cells (hiPSCs), the interest in HD-MEAs is rapidly growing, as such devices are suitable to assess hiPSC functionality of healthy and disease phenotypes. In this study, we used a 26,400-electrode CMOS chip (Ballini et al., 2014), with a sensing area of $3.85 \times 2.10 \mathrm{~mm}^{2}$, an electrode pitch of $17.5 \mu \mathrm{m}$ and an electrode size of $5 \times 9 \mathrm{\mu m}^{2}$, which provided subcellular resolution for readout and stimulation. The device enabled targeting of the axon initial segment (AIS) for stimulation, which was demonstrated to ensure efficient and accurate stimulation (Radivojevic et al., 2016; Bakkum et al., 2018), and the device enabled signal readout upon stimulation in direct proximity to the cell soma of the very same cell.

An important issue with electrical stimulation through microelectrodes in densely packed arrays is the so-called "stimulation artifact," which is characterized by saturation of the recording amplifiers that are connected to the stimulation electrode itself and the surrounding electrodes during hundreds of microseconds or longer. This saturation is a consequence of the large stimulation signal amplitudes, $\sim 50-100 \mathrm{mV}$, while the readout amplifiers feature microVolt sensitivity. Different from other approaches (Hottowy et al., 2012), we did not aim at recovering the signal on the stimulation electrode itself, as the large density of electrodes, present in our array, allows for recording from different electrodes, still spanning under the same neuron of interest. Moreover, we did not use any strategy to suppress artifacts in this study, as our interest was to compare the different stimulation strategies and parameters also with respect to artifact generation. Electrodes at a distance of only $17.5 \mu \mathrm{m}$ from the stimulation electrode were already available for readout in all cases, partially due to the comparably low stimulationsignal amplitudes that we could afford owing to accurate targeting of the stimulation-sensitive AIS (Radivojevic et al., 2016). The artifact depends on the stimulation signal amplitude, the applied waveform, and its duration. Therefore it is crucial to identify the stimulation signal that produces the lowest artifact while still reliably inducing an action potential (AP).

This study was targeted at finding optimal stimulation modalities, i.e., to achieve the most efficient stimulation of neurons with our HD-MEAs at minimal artifacts, by comparing different stimulation waveforms, amplitudes and durations, both in voltage and current mode. We used biphasic and monophasic rectangular waveforms for stimulation in voltage mode (Wagenaar et al., 2004), and charge-balanced biphasic and triphasic rectangular waveforms for stimulation in current mode (Wagenaar et al., 2004; Hottowy et al., 2012; Grosberg et al., 2017). We compared the efficacy of the voltage and current stimulation regimes, characterized the influence of the electrode impedance, and measured stimulability during cell growth and development in culture. Finally, we simulated and tested different multi-electrode configurations and compared the obtained results.

\section{MATERIALS AND METHODS}

\section{Animal Use}

All experimental protocols were approved by the Basel Stadt veterinary office according to Swiss federal laws on animal welfare and were carried out in accordance with the approved guidelines.

\section{High-Density Microelectrode Arrays}

A CMOS-based HD-MEA (Ballini et al., 2014) was used for in vitro stimulation and recording. The device features $26,400 \mathrm{Pt}$ electrodes (each $5 \times 9 \mu \mathrm{m}^{2}$ at a pitch of $17.5 \mu \mathrm{m}$ ) occupying a sensing area of $3.85 \times 2.10 \mathrm{~mm}^{2}$. The HD-MEA system includes 1,024 configurable readout channels that can be used to record simultaneously. The readout-channel noise is $2.4 \mu \mathrm{V}_{\mathrm{rms}}$ 
in the band between $300 \mathrm{~Hz}$ and $10 \mathrm{kHz}$ and $5.4 \mu \mathrm{V}_{\mathrm{rms}}$ in the band between 1 and $300 \mathrm{~Hz}$. The readout channels' gain is programmable up to $78 \mathrm{~dB}$, depending on the application. Additionally, the device features 32 stimulation units that can be used in both, current or voltage mode. The sampling frequency is $20 \mathrm{kSamples} / \mathrm{s}$, and the overall power consumption is $75 \mathrm{~mW}$. Gold bond wires connect the chips to printed circuit boards (PCBs) and are protected from saline solutions (e.g., culture medium) using epoxy (Epo-Tek 353ND, 35ND-T, Epoxy Technology Inc., Billerica, MA, United States). The electrodes were coated with Pt-black, the chips were then sterilized for $40 \mathrm{~min}$ in $70 \%$ ethanol and rinsed 3 times with deionized (DI) water before every cell plating.

\section{Platinum Black Deposition}

A porous Pt-black layer was deposited on the electrodes to increase the surface area and decrease the electrode impedance, which improves the signal-to-noise ratio (SNR) of recorded signals. A $2 \mathrm{~mL}$ solution of chloroplatinic acid hexahydrate (7 mM, Sigma-Aldrich, Saint Louis, MO, United States) and lead acetate $(0.3 \mathrm{mM}$, Honeywell, Morris Plains, NJ, United States) in DI water was pipetted onto the exposed region of the HDMEA chip. A Pt reference electrode was immersed in the solution and current of $550 \mu \mathrm{A}$ was applied to the array electrodes for $1.30 \mathrm{~min}$.

\section{Cell Cultures}

Prior to culturing cells, the HD-MEA electrode area was treated with $20 \mu \mathrm{L}$ of $0.05 \%$ (v/v) poly(ethyleneimine) (Sigma-Aldrich) in borate buffer (Thermo Fisher Scientific, Waltham, MA, United States) at $8.5 \mathrm{pH}$, for $40 \mathrm{~min}$ at room temperature. This step improves cell adhesion and makes the substrate more hydrophilic. We rinsed the chips three times with DI water. Next, we added $8 \mu \mathrm{L}$ of $0.02 \mathrm{mg} \mathrm{ml}^{-1}$ laminin (Sigma-Aldrich) in Neurobasal medium (Gibco, Thermo Fisher Scientific) to support the growth and differentiation of the cells. The chips were incubated with laminin for $30 \mathrm{~min}$ at $37^{\circ} \mathrm{C}$. During this time, we dissociated cortices of Wistar rats at embryonic day 18 in trypsin with $0.25 \%$ EDTA (Gibco). After 20 min of digestion, the cortices were washed twice with plating medium, then triturated, and the cells were counted. We counted with a hemocytometer by diluting the cells in $0.4 \%$ Trypan blue stain solution (Gibco). We then seeded between 15,000 and 25,000 cells over an active area of approx. $8 \mathrm{~mm}^{2}$. The chips were afterward incubated at $37^{\circ} \mathrm{C}$ for $30 \mathrm{~min}$ before adding $1.5 \mathrm{~mL}$ of plating medium. The plating medium consisted of $450 \mathrm{~mL}$ Neurobasal (Invitrogen, Carlsbad, CA, United States), $50 \mathrm{~mL}$ horse serum (HyClone, Thermo Fisher Scientific), $1.25 \mathrm{~mL}$ Glutamax (Invitrogen), and $10 \mathrm{~mL} \mathrm{~B}-27$ (Invitrogen). After $76 \mathrm{~h}$, we changed $50 \%$ of the plating medium to growth medium, which consisted of $450 \mathrm{~mL}$ D-MEM (Invitrogen), $50 \mathrm{~mL}$ Horse Serum (HyClone), $1.25 \mathrm{~mL}$ Glutamax (Invitrogen) and $5 \mathrm{~mL}$ sodium pyruvate (Invitrogen). The procedure was repeated twice a week. The chips were kept inside an incubator at $37^{\circ} \mathrm{C}$ and $5 \% \mathrm{CO}_{2}$. Every chip was equipped with a lid, and additional DI water in a $35-\mathrm{mm}-\varnothing$ petridish was added to prevent evaporation. All the experiments were conducted between days in vitro (DIVs) 10 and 30.

\section{Microscopy and Stainings}

We used NeuroFluor NeuO (Stemcell Technologies, Vancouver, Canada) live staining to locate neurons on the array before the stimulation experiments. The cells were incubated for $1 \mathrm{~h}$ at $37^{\circ} \mathrm{C}$ with $2 \mathrm{~mL}$ growth medium containing $0.15 \% \mathrm{NeuO}$. The chips were then washed 2 times with growth medium.

We also performed neuron fixation after stimulation experiments by using 4\% paraformaldehyde (Life Technologies, Thermo Fisher Scientific). Sample permeabilization and blocking of non-specific antibody binding were done using a PBS $1 \times$ solution containing: 10\% normal donkey serum (NDS) (SigmaAldrich), $1 \%$ bovine serum albumin (BSA) (Sigma-Aldrich), $0.02 \% \mathrm{Na}-\mathrm{Az}$ (Sigma-Aldrich), 0.5\% Triton X (Sigma-Aldrich). Primary and secondary antibodies were diluted in a PBS $1 \times$ solution containing: 3\% normal donkey serum (NDS), $1 \%$ bovine serum albumin (BSA), $0.02 \% \mathrm{Na}-\mathrm{Az}, 0.5 \%$ Triton $\mathrm{X}$. We used antibodies against MAP2 (Abcam, Cambridge, United Kingdom), Ankyrin G (Santa Cruz Biotechnology, Dallas, TX, United States), and the fluorescent dye Hoechst (Invitrogen) to stain neurons, axonal initial segments (AIS), and nuclei. We imaged cells on the HD-MEA chip with a Nikon NiE upright confocal microscope, with a Yokogawa W1 spinning disk scan head, 6 laser lines and a fluorescence recovery after photobleaching (FRAP) unit.

\section{Stimulation and Data Analysis}

Electrical stimulation was controlled via an on-chip digital analog converter (DAC) and software programmable through a Python application programming interface (API).

We used both, voltage and current stimulation modalities. In both, a charge is applied to the stimulation electrode. Ideally, only charge redistribution in the double-layer capacitor, formed at the electrode/electrolyte interface, occurs and charge transfer and redox reactions involving electron transfer at the electrode surface (Faradaic processes) are avoided. Using current stimulation, the charge can directly be controlled, while the voltage may assume large values depending on the specific current path. High electrode voltages may produce unwanted electrochemistry (Faradaic processes), tissue damage, or electrode degradation. In the case of voltage stimulation, one can control the voltage, while the injected current depends on the electrode impedance (Supplementary Figure 1), which may vary considerably due to fabrication variation or aging. Precisely controlling the applied voltage helps to prevent electrolysis, which may occur outside the water window and may damage the electrodes or cause cell death.

We used a randomized voltage stimulation protocol including four different waveforms: biphasic cathodic-anodic, biphasic anodic-cathodic, monophasic anodic, monophasic cathodic, see also Figure 2A (Wagenaar et al., 2004). The protocol included four durations of 50,100,150, and $200 \mu$ s per phase and six stimulation signal amplitudes (40, 80, 120, 160, 200, and $240 \mathrm{mV}$ peak-to-peak). For current stimulation, we applied a randomized protocol of two waveforms, biphasic anodiccathodic and triphasic anodic-cathodic-anodic, both charge balanced (Grosberg et al., 2017), five durations of 10, 15, 18, 20, 
and $50 \mu \mathrm{s}$ per phase, and eight stimulation signal amplitudes $(42,63,84,105,126,147,168$, and 189 nA). Every individually shaped stimulation pulse of both modalities was repeated 30 times during the entire protocol in a randomized way in order not to evoke neuronal plasticity processes. The stimulation frequency was $1 \mathrm{~Hz}$ for both modalities, as stimulation in the frequency band between 0.2 and $1 \mathrm{~Hz}$ was reported not to entail significant changes in the AIS position (Grubb and Burrone, 2011). We selected $1 \mathrm{~Hz}$, the upper bound, to limit overall time needed for the stimulation experiments.

A custom-made software was used to visualize and record the extracellular signals from the electrodes. The extracellular action potential (EAP) spatial distribution or "electrical footprint" of a neuron, which is the voltage-signal distribution over the multiple electrodes, was reconstructed using spike sorting algorithms (UltraMegaSort, Hill et al., 2011). The software identifies the spikes with a threshold of 4.5 times the standard deviation of the noise. Using this software, we then could identify and select the stimulation electrodes based on the EAP amplitudes.

The collected data was analyzed in MATLAB. To verify the presence of an evoked APs, we set a threshold of four times the standard deviation of the noise, together with a temporal window of $1.5 \mathrm{~ms}$. An automatic script registered the EAPs for the 30 repetitions of every sent waveform, and rendered a visual record that could be inspected to verify the counting. Cases where the artifact partially covered the EAPs were classified as "missing EAP" during automatic registration. In these cases, we applied visual inspection and manual correction as appropriate.

\section{Impedance Characterization}

To characterize electrode impedances, we applied a readout gain of 2 and 20 repetitions of a biphasic anodic-cathodic current stimulation pulse with a duration of $1 \mathrm{~ms}$ per phase and an amplitude of $140 \mathrm{nA}$ to bright Pt electrodes and with a duration of $2.5 \mathrm{~ms}$ per phase and an amplitude of $560 \mathrm{nA}$ to Ptblack electrodes. Such low gain in the readout channels avoided DC voltage saturation of the stimulation channel. The lower waveform durations and amplitudes avoided channel saturation in case of bright $\mathrm{Pt}$, because of the higher impedance.

To determine the electrode impedance, we fitted the voltage readout on the stimulation electrode with equations derived from the Gouy-Chapman-Stern model of an electrode (Franks et al., 2005) (Supplementary Figure 2). We added a constant equivalent input impedance $Z_{\text {in }}$ for the recording channel input impedance in parallel to the electrode equivalent circuit. The electrode equivalent circuit had two unknown values, the charge transfer resistance, $R_{\mathrm{ct}}$, and the double layer capacitance, $C_{\mathrm{dl}}$. These values were computed by fitting the obtained experimental data in MATLAB using the following equation:

$$
V(t)=\frac{I_{\mathrm{stim}} R_{\mathrm{ct}}\left(1-e^{-\frac{t}{C_{\mathrm{dl}} R_{\mathrm{ct}}}}\right)}{1+\frac{R_{\mathrm{ct}}}{Z_{\mathrm{in}}}\left(1-e^{-\frac{t}{\mathrm{C}_{\mathrm{dl}} R_{\mathrm{ct}}}}\right)}
$$

In this equation $V$ is the readout voltage and $I_{\text {stim }}$ the applied stimulation current. We defined initial values for $C_{\mathrm{dl}}$ and $R_{\mathrm{ct}}$ by referring to values reported in literature (Héduit et al., 1996; Franks et al., 2005; Oldham, 2008; Joye et al., 2009; Sharma and Bhatti, 2010).

To confirm the simulation results with a larger number of electrodes, we applied a sine-wave voltage stimulation on the reference electrode surrounding the array and recorded the corresponding signals through electrode sets of the array. The sine wave had a frequency of $1 \mathrm{kHz}$ and an amplitude of $50 \mathrm{mV}_{\mathrm{pp}}$ (peak-to-peak). The recording channels and circuits are characterized by a finite and constant input impedance, which should ideally be high for neural applications and higher than the electrode impedance to ensure signal integrity (Obien et al., 2015). We compared the obtained electrode impedances with the input impedances of the recording channels for the whole array to characterize impedance homogeneity across the array. We also performed impedance measurements on three stimulation electrodes in PBS, before and after conducting a full set of stimulation experiments in current and voltage mode. After the experiment, the impedance varied in average by $0.07 \mathrm{nF}(\sim 5 \%$ of the average electrode impedance value for Pt black).

\section{COMSOL Simulations}

We simulated the stimulation pulse extension across the array of the used multi-electrode configurations in COMSOL Multiphysics 5.3a. The model includes 4 main components (libraries): geometry, materials, electric currents, and mesh. For the geometry, we used a configuration of 36 planar electrodes, with a $5 \times 9 \mu \mathrm{m}^{2}$ surface area for each electrode and a pitch of $17.5 \mu \mathrm{m}$, which is consistent with the HD-MEA electrode characteristics. An external block of $500 \times 500 \times 100 \mu \mathrm{ml}$ was added as the electrolyte solution. Four reference electrodes

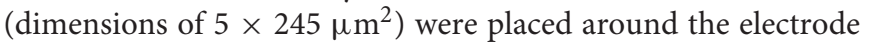
array. The electrodes were simulated assuming platinum as electrode material, while the electrolyte solution was a saline solution with an electrical conductivity of $0.7 \mathrm{~S} \mathrm{~m}^{-1}$. For the electrical characterization, we simulated voltage stimulations. We used a biphasic anodic-cathodic waveform with an amplitude of $100 \mathrm{mV}$ and a duration of $100 \mu \mathrm{s}$ per phase. The electriccurrent library was used to simulate the voltage and electric field distributions upon voltage stimulation.

\section{Multi-Electrode Stimulation}

To implement multi-electrode stimulations in voltage mode, we used a custom-made Python script to control two DACs to stimulate two electrodes at the same time. In a first configuration, we applied a biphasic waveform on one electrode and the same waveform but with opposite sign on a neighboring electrode. This configuration limited the charge flow across the array, which decreased the artifact extension and spreading. In a second configuration, we applied a biphasic waveform to one electrode and connected the neighboring electrode to ground with the intention to limit the electric field and artifact extension. External reference electrodes remained always connected.

\section{Availability of Materials}

Adapted MATLAB scripts and COMSOL model scripts, used for the analysis of the data in Figures 2-6 are available at the 
following repository: ElectricalStimulation (link: https://github. com/sronchi/ElectricalStimulation). Moreover, we can provide raw data sets (total of $10 \mathrm{~TB}$ ) at reasonable request.

\section{RESULTS}

\section{Artifacts of Current and Voltage Stimulation}

A major limitation of any electrical stimulation is the resulting artifact, which obscures the EAP readout likewise in current and voltage modes. To compare artifacts generated during voltage and current stimulation of cortical neurons, we plated 15,000 cortical neurons on the array and labeled them neurons with NeuroFluor NeuO live-staining (Figure 1A) to identify individual neurons. We determined the most suitable electrode and the smallest stimulation signal amplitude that could evoke EAPs with $90 \%$ success rate over 30 repetitions. We used biphasic waveforms in both modalities and compared cases with similar artifact shapes. The duration was set to 100 and $20 \mu \mathrm{s}$ per phase for voltage and current stimulation, respectively. The different durations were a consequence of the high efficiency of the stimulation buffers in current mode, which showed a reliable charge injection for durations longer than $18 \mu \mathrm{s}$ (Supplementary Figures 3, 4). In current mode, the stimulation buffers could deliver a sharp charge injection regardless of the electrode impedance. In voltage mode, however, the shortest efficient pulse duration was found to be $>50 \mu$ s (Paragraph 3.2, Supplementary Figure 4), due to the different stimulation buffers' design (Ballini et al., 2014).

Since the artifact duration is governed primarily by the stimulation pulse duration, shorter current pulses produced shorter, more easily detectable and distinguishable AP artifacts than the voltage pulses. This is shown in Figures 1B,C. For the latter, not only were the nearby extracellular APs obscured, but the artifact amplitudes were also significantly larger. In voltage mode, the evoked EAPs were shifted with respect to the baseline, in comparison to spontaneous EAP activity, so that they could not always be easily detected by using a negative amplitude threshold (four times the standard deviation of the noise) for spike detection. Only the neurons producing high-amplitude EAPs could be detected by using a negative voltage threshold while the other neurons were not used for analysis.

Owing to the high-density electrodes, the short current stimulation pulse enabled a signal readout and determination of stimulation success already on the next neighboring electrode, $17.5 \mu \mathrm{m}$ away from the stimulation site (Figures 1B,C, center and right). Upon stimulating axonal compartments, like the AIS, it was possible to measure signals at the corresponding cell soma of the same neuron, which enabled to unambiguously determine stimulation success.

\section{Effect of Durations, Amplitudes and Waveforms, in Voltage and Current Stimulation Modes}

Although we noticed that the HD-MEA produced smaller artifacts in current mode, we wanted to study the relevant parameters to efficiently evoke APs in both, current and voltage modes. Current stimulation is preferred and used for many in vivo applications (Raspopovic et al., 2014), due to the fact that the injected charge can be determined independently of the impedance. However, voltage stimulation offers the advantage to precisely control the voltage and avoid electrode or cell/tissue damage as a consequence of electrolysis (for a more detailed discussion see Section 2.6). In line with previous electricalstimulation studies in vitro (Wagenaar et al., 2004; Hottowy et al., 2012; Grosberg et al., 2017), we investigated different parameters for both, current and voltage modes. Our strategy was to efficiently stimulate neurons in the region of the AIS and to then read out the corresponding evoked action potentials at the cell soma and several other locations, which was possible due to the availability of a large number of electrodes at high density, the small signal amplitudes needed to stimulate at the AIS, and the possibility to deliver short and efficient stimulation pulses $(\geq 18 \mu \mathrm{s})$. For voltage stimulation, we used biphasic cathodic-anodic, biphasic anodic-cathodic, monophasic anodic and monophasic cathodic waveforms. The stimulation protocol included four durations for every waveform and six amplitudes in a randomized sequence $(40,80,120,160,200$, and $240 \mathrm{mV}$ peak to peak). Neurons were stimulated between DIV 10 and 30. Stimulation electrodes were selected after identifying the spatial distribution or electrical footprint of extracellular EAPs of individual neurons. Electrodes recording the highest amplitudes of single-neuron action potentials were selected as stimulation electrodes, as they were presumably located under the neuronal compartments that are most sensitive to stimulation (Radivojevic et al., 2016; Bakkum et al., 2018). The compartment producing the largest-amplitude EAPs and being most sensitive to stimulation has been recently identified as the AIS (Radivojevic et al., 2016; Bakkum et al., 2018). The results are displayed in Figure 2A: The stimulation results of two different neurons upon applying four stimulation voltage waveforms with different amplitudes and waveform durations are shown. The monophasic cathodic waveform was found to be the most efficient waveform in evoking APs in the voltage mode, followed by the biphasic anodic-cathodic waveform. The monophasic anodic and the biphasic cathodic-anodic waveform featured lower efficiency. Another result we observed is that a phase duration of $50 \mu \mathrm{s}$ was not sufficient to reliably evoke APs, whereas there was no major difference for phase durations longer than $100 \mu$ s. In Figure 2B, recorded voltage waveforms and close-ups of successful (AP was elicited, black) and unsuccessful (no AP, green) stimulations are shown to demonstrate how EAPs look like in the presence of stimulation artifacts. To consolidate the stimulation results in $2 \mathrm{~A}$, we repeated the randomized protocols with 16 additional neurons (Figure 2C). We determined the peak-to-peak voltage $\left(V_{p p}\right)$, which was needed to evoke APs in $90 \%$ of the stimulations over 30 repetitions for those cells. Two phase durations, $50 \mu \mathrm{s}$ (gray) and $100 \mu \mathrm{s}$ (green), were used and compared. At the top of the graph, the percentages of failure in evoking APs, while using the 4 waveforms up to a maximum amplitude of $240 \mathrm{mV}$ are given for the 2 different durations. The obtained results confirmed the aforementioned low efficacy of $50 \mu$ s phase duration. For using a phase duration of $50 \mu \mathrm{s}$, one should, for a successful stimulation, deliver the same charge as for using $100 \mu \mathrm{s}$, but the settling time 


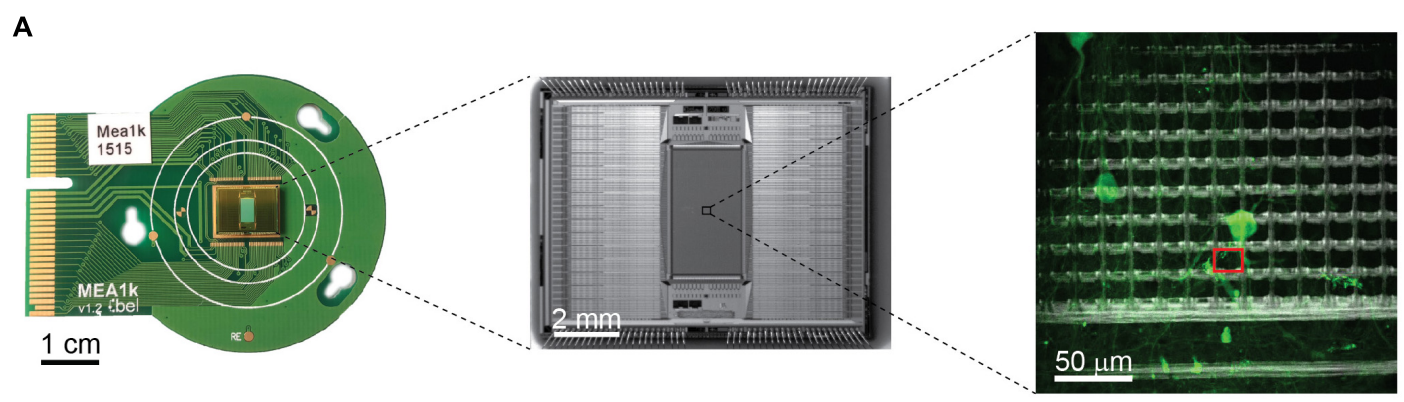

B

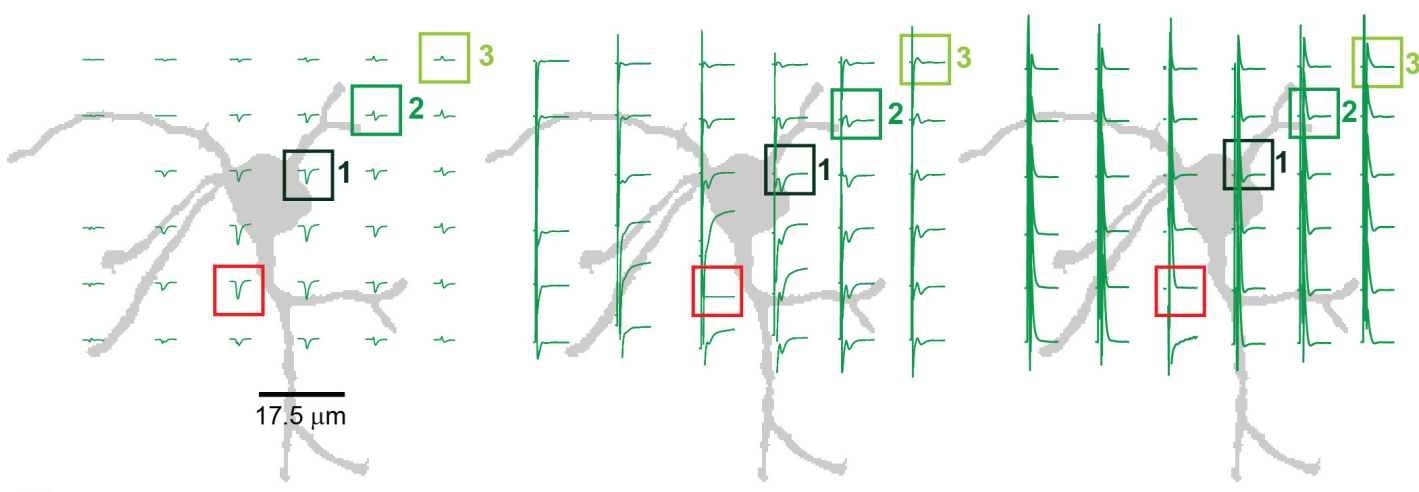

Stimulation electrode

C

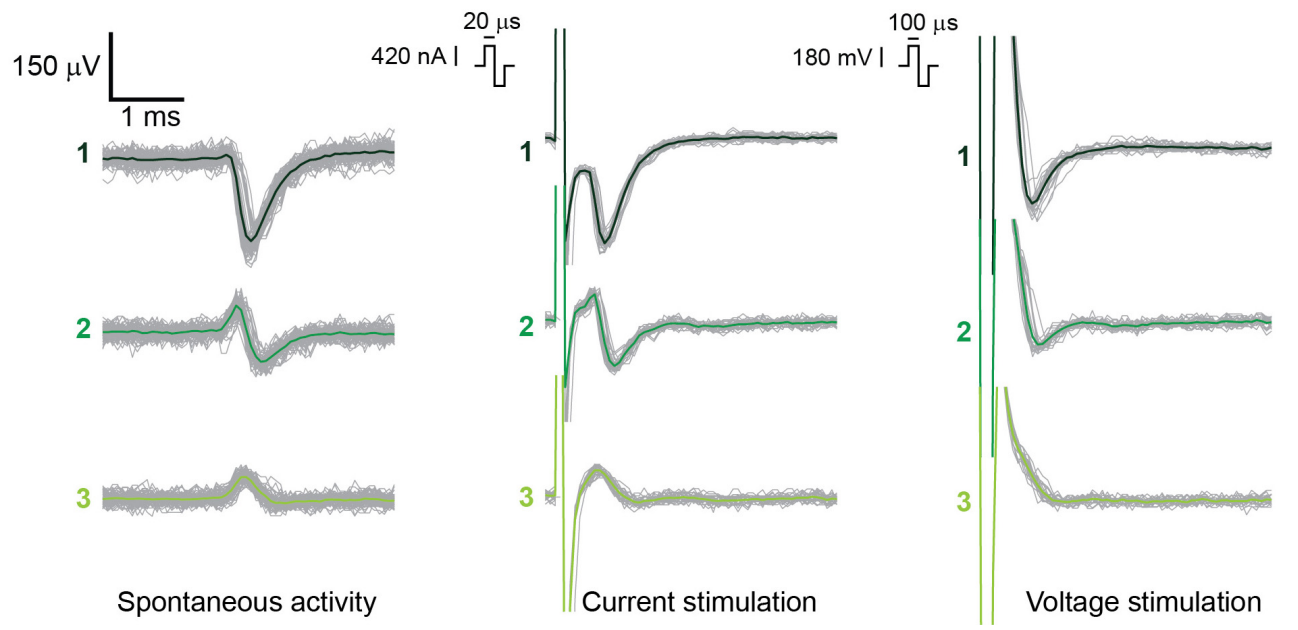

FIGURE 1 | (A) (from left to right) PCB-mounted HD-MEA chip, photomicrograph of the chip, and enlargement of a subsection of the array including about 100 electrodes with live-stained neurons highlighted in green. The stimulation electrode used in panel $\mathbf{B}$ is highlighted in red. The picture was taken using an immersion $60 \mathrm{x}$-magnification lens at the periphery of the array, where the cell density is lower. (B) Illustration of the neuron, labeled in panel $\mathbf{A}$, and corresponding electrode locations, with superimposed measured signals. (Left) Spontaneous EAPs obtained after spike sorting. (Center) EAPs after current stimulation of the selected neuron. (Right) EAPs after voltage stimulation. Current stimulation entailed a biphasic anodic-cathodic waveform of $20 \mu \mathrm{s}$ per phase. Voltage stimulation entailed a biphasic anodic-cathodic waveform of $100 \mu$ s per phase. The smallest stimulation signal amplitudes that still evoked APs 27 times during 30 repetitions (90\% efficiency) were used. (C) Signals from three of the readout electrodes in panels $\mathbf{A}$ and $\mathbf{B}$ (numbered boxes). (Left) Extracellular signals recorded during spontaneous neuronal activity composed of $>100$ detected EAPs. (Center and right) Extracellular signals recorded from the same electrodes during 30 repetitions of current and voltage stimulation. 

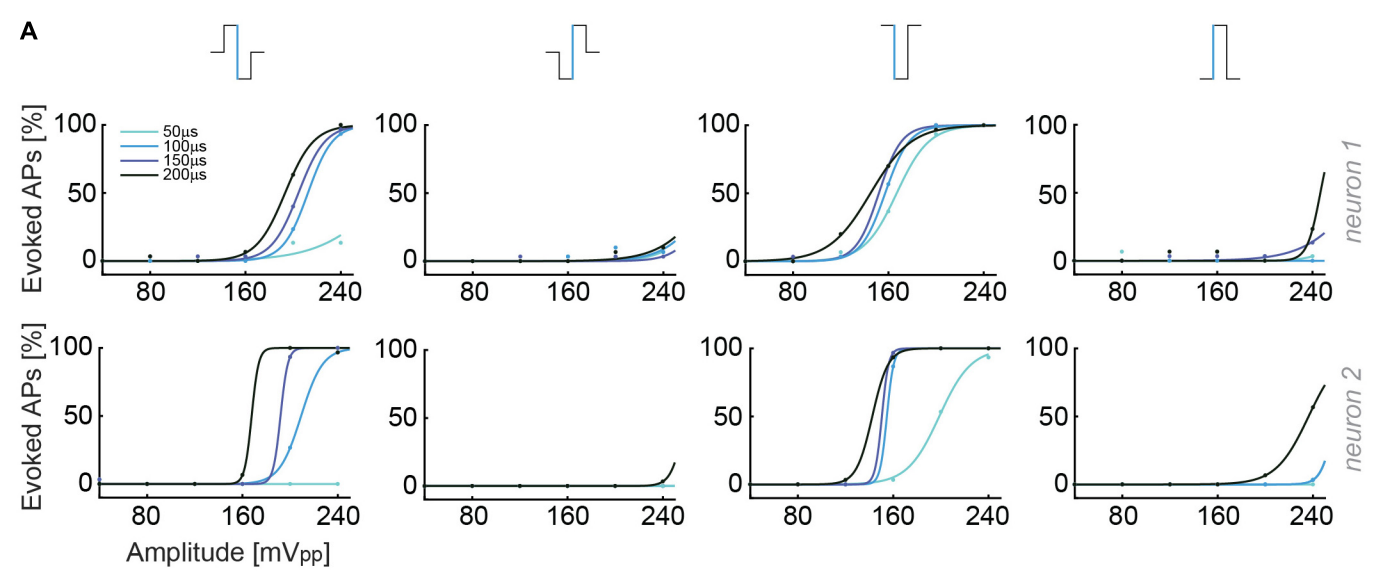

B

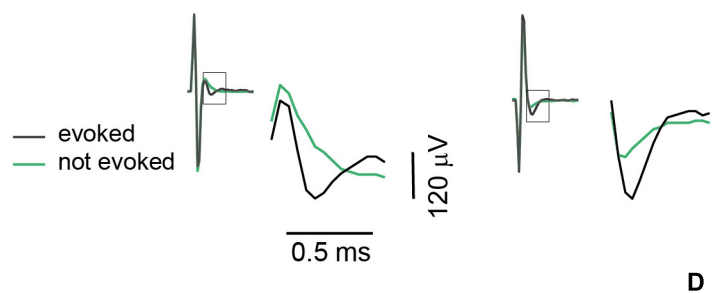

C
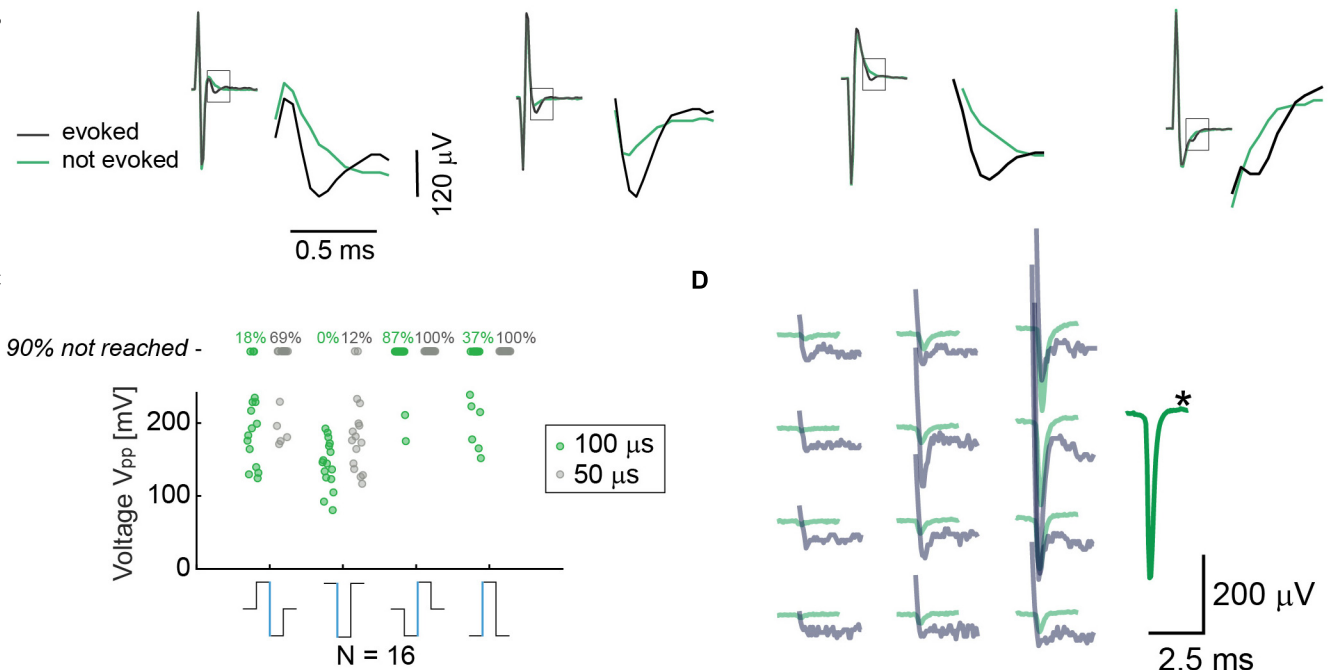

E
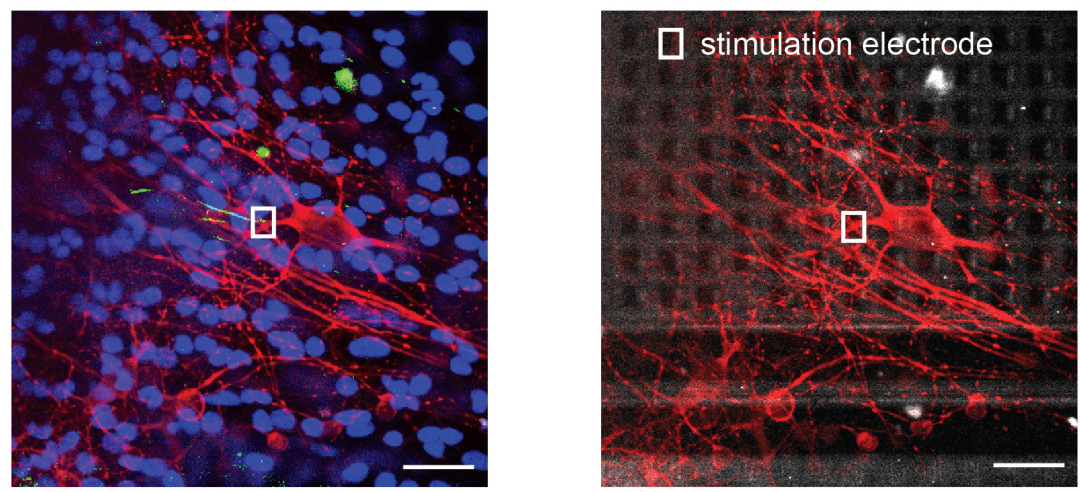

FIGURE 2 | (A) Results from voltage stimulation of two neurons. At the top, the four different waveforms that were used are displayed. The figure shows that lower voltage amplitudes are sufficient to evoke APs when using monophasic cathodic and biphasic anodic-cathodic waveforms. A phase duration of $50 \mu s$ was less efficient than phase durations of 100, 150, and $200 \mu \mathrm{s}$. We applied 30 repetitions for every waveform, duration and amplitude in a randomized manner.

(B) Recorded voltage signals including stimulation artifact for successful and non-successful voltage stimulations are displayed for the four stimulation waveforms in panel A. No measures were taken to suppress the artifact. The close-ups show the region, in which the neuron's response eventually occurred. The voltage signal (artifact only) recorded during/after a stimulation that did not evoke an AP is displayed in green, voltage signals (artifact plus superimposed neuronal response) recorded during a successful stimulation are displayed in black. (C) Voltage stimulation results of 16 neurons. The points represent the smallest voltages (Vpp) that evoked APs in $90 \%$ of the stimulations during 30 repetitions; the signal duration was $100 \mu \mathrm{s}$ per phase (green dots) or $50 \mu \mathrm{s}$ per phase (gray dots). At the top, the percentages of failure in evoking APs upon using the 4 waveforms with amplitudes of up to $240 \mathrm{mV}$ are given for the 2 different durations. (D) Overlay of the spike-sorted spontaneous activity of a neuron (green) and its response (gray) upon voltage stimulation through the electrode marked with a black star $(*)$. (E) Neuron stainings; nuclei were stained in blue (Hoechst), neuronal structures in red (anti-MAP2) and the AIS in green (Ankyrin G). The stimulation electrode is indicated with a rectangle (white). In the right picture, the electrode array is visible below the stimulated neurons. Scale bar: $35 \mu \mathrm{m}$. 
of the stimulation buffers and the electrode impedance imposed limits on the stimulation efficacy with such short phase durations (see Supplementary Figure 4).

To confirm that the selected neurons were effectively stimulated, we superimposed the "electrical footprints" of spontaneous activity after spike sorting (using UltraMegaSort) with the spatial distribution of stimulation-evoked EAPs. The spontaneous activity was recorded using a high-density block of electrodes in the region of interest during at least 1 min (>100 EAP). The superposition shows a spatial and temporal match of spontaneous and stimulation-triggered EAPs. However, the amplitudes of the superimposed EAPs are not so easy to compare as a consequence of the stimulation artifact (Figure 2D). A clear identification of the neuron could be performed by using its electrical "footprint," the spatial distribution of extracellular APs in conjunction with upright confocal microscopy. Using these features and methods, we could prove that, indeed, the neuron of interest was stimulated in its perisomatic region as EAP readout of the very same neuron was possible, e.g., in an axonal branch nearby. Stimulation was very selective, and individual neurons could be stimulated without eliciting EAPs in neighboring neurons.

After execution of the electrical stimulation protocols, selected neurons were stained for correlating neuron morphologies with their EAP spatial distribution, recorded through the electrodes. In particular, we investigated which neuronal compartment was closest to the stimulation electrode. We observed that the most efficient stimulation electrode was located in close proximity to the AIS (green AnkyrinG staining), confirming previous reports (Radivojevic et al., 2016; Bakkum et al., 2018) (Figure 2E).

In a second set of experiments, we used similar protocols for the current stimulation mode, where we investigated the effect of two different waveforms, namely biphasic anodic-cathodic, and triphasic anodic-cathodic-anodic (amplitude ratio 2:3:1). We used five phase durations and eight signal amplitudes in a randomized sequence $(42,63,84,105,126,147,168$, and $189 \mathrm{nA})$. Monophasic waveforms were not used to not compromise charge balancing. The results of two different stimulated neurons in Figure 3A show that the two waveforms provide similar efficiency in stimulating the targeted neurons for different phase durations and amplitudes. In Figure 3B, recorded voltage waveforms and close-ups of successful (AP was elicited, black) and unsuccessful (no AP, red) stimulations are shown to demonstrate how EAPs look like in the presence of stimulation artifacts. The results can be compared with panel $2 \mathrm{~B}$, showing the signals for the voltage stimulation mode. For the voltage stimulation mode, the AP always is superimposed on the artifact, whereas, in current mode, the evoked EAP is temporally more clearly separated from the artifact and much easier to detect. This better detectability is, to some extent, a consequence of the shorter duration of the stimulation signal in the current mode. To consolidate the stimulation results, we repeated the same randomized protocol with 20 additional neurons with a phase duration of $20 \mu \mathrm{s}$. Several HD-MEAs were used in parallel to speed up experiments. The obtained results are shown in
Figure 3C. All current stimulation events delivering charges of up to $3 \mathrm{pC}$ were successful.

As in the case of voltage stimulation, an overlay of the spatial distribution of spontaneous-activity EAP signals and the current stimulation-induced EAP was used to confirm the identity and successful stimulation of the targeted neurons. Spontaneous and stimulated EAPs match temporally, spatially and amplitude-wise (Figure 3D). Additionally, readout electrodes very close to the stimulation electrode also provided clearly detectable signals. The result in Figure 3D can be compared to Figure 2D for voltage mode, where an amplitude comparison was not possible as a consequence of the large artifact.

To summarize, the most efficient stimulation in voltage mode can be achieved by using monophasic cathodic and biphasic anodic-cathodic waveforms with a duration of $100 \mu$ s per phase. In current mode, biphasic and triphasic waveforms show the same efficacy, but the biphasic waveform is shorter than the triphasic waveform, which facilitates EAP readout. Durations of 18-20 $\mu$ s per phase have proven to be efficient (Supplementary Figure 3). Additionally, we noticed that artifact amplitudes are larger for monophasic voltage stimulation and biphasic current stimulation waveforms. In the case of the commonly used voltage stimulation mode, a biphasic anodic-cathodic waveform should be used, as it represents a good combination of stimulation efficacy and artifact duration.

\section{Impedance Measurements to Compare Voltage and Current Stimulation}

As mentioned in Section 3.2, both voltage and current stimulation are widely used to stimulate neuron. Voltage stimulation offers the advantage to reliably obviate Faradaic processes by precisely controlling the electrode voltage and keeping it significantly below $0.8-1 \mathrm{~V}$ to obviate water electrolysis and cell and electrode damage (Weiland et al., 2002; Wagenaar et al., 2004). However, the injected charge cannot be controlled and is a function of the electrode impedance. On the other hand, the voltage cannot be controlled upon using current stimulation, so that high electrode voltages can occur in case of high impedance, which may entail unwanted electrochemistry, tissue damage, or electrode degradation. Yet, the charge delivered by the electrode (not the charge path in the preparation) can be precisely controlled, and potentially short current stimulation durations entail short artifacts and fast recovery to baseline values.

To better compare efficacies and differences of current- and voltage-controlled stimulation modes in depolarizing neuronal membranes (from -70 to $-55 \mathrm{mV}$ ) and evoking APs, it is necessary to also consider the delivered charge. To this end, we established a method to determine electrode impedances, so that charge injection of voltage and current pulses could be calculated and compared.

We applied a biphasic current pulse to an electrode and used a low gain $(G=2)$ for reading out the voltage signals of the same electrode (see Paragraph 2.7). We then fitted the voltage readout from the stimulating electrode with a theoretical electrode model (Supplementary Figure 2 and Figure 4A) by using only the first half of the biphasic anodic-cathodic waveform. The 


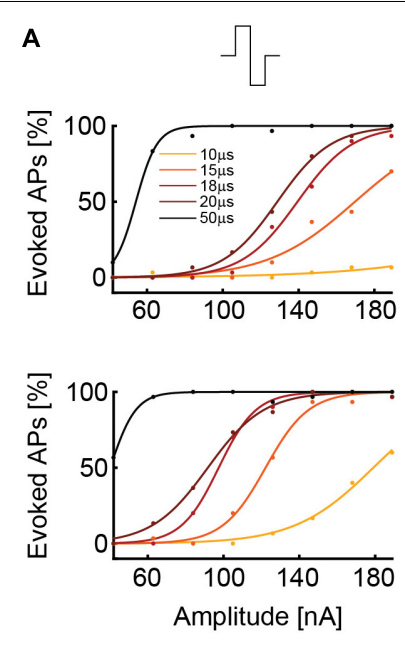

C

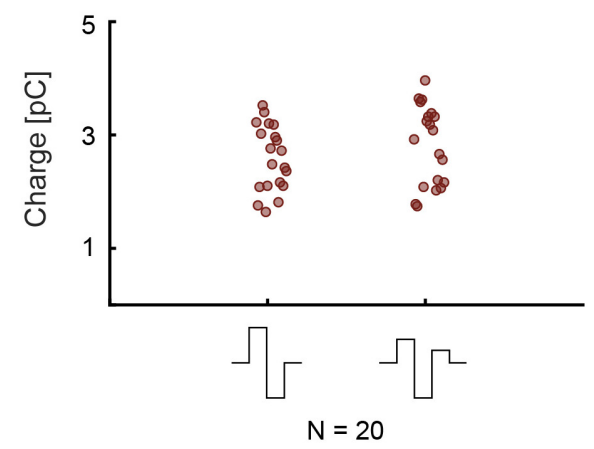

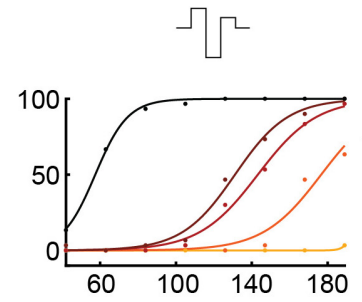

.

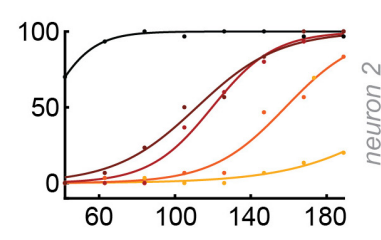

D

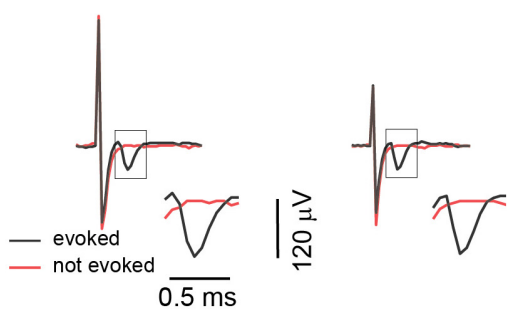

FIGURE 3 | (A) Results from current stimulation of two neurons. At the top, the two waveforms that were used are displayed. The efficacy in evoking APs is almost the same for both waveforms. 30 repetitions were used for every waveform, phase duration and stimulation signal amplitude in a randomized manner. (B) Recorded voltage signals including stimulation artifacts for successful and non-successful current stimulations are displayed for the two stimulation waveforms in panel A. No measures were taken to suppress the artifact. The close-ups show the region, in which the neuron's response eventually occurred. The voltage signal (artifact only) recorded during/after a stimulation that did not evoke an AP is displayed in red, voltage signals (artifact plus superimposed neuronal response), recorded during a successful stimulation, are displayed in black. (C) The current stimulation results of 20 neurons are displayed. The points represent the smallest charges that evoked APs in $90 \%$ of the stimulations during 30 repetitions; the signal duration was $20 \mu \mathrm{s}$ per phase. (D) Overlay of the spike-sorted spontaneous activity of a neuron (red) and its response (gray) upon current stimulation through the electrode marked with a black star $\left(^{*}\right)$.

charge transfer resistance, $R c t$, and the double layer capacitance, $C d l$, were kept as unknown values. After examining $(n=10)$ bright Pt and Pt-black electrodes, we found that the $C d l$ was $0.077 \pm 0.0138 \mathrm{nF}$ for bright Pt electrodes and $1.44 \pm 0.15 \mathrm{nF}$ for Pt-black electrodes (Figure 4B). The results were obtained with an electrode area of $5 \times 9 \mu \mathrm{m}^{2}$.

To extend these results to a larger number of electrodes, we applied a sine-wave stimulation to 26,400 electrodes and proved that their impedance (in terms of voltage readout) was homogeneous over the array (see histograms in Supplementary Figure 5). This allowed us to use the mean capacitance value of $1.44 \mathrm{nF}$ for Pt-black to compare current and voltage stimulation. Based on the obtained capacitance values, we calculated the charge associated with voltage stimulation and compared it to current stimulation for the same neuron (Figure 4C). We used biphasic waveforms in both modalities, with a duration of $20 \mu \mathrm{s}$ per phase in current mode and $100 \mu \mathrm{s}$ per phase in voltage mode. We used a randomized stimulation protocols including 30 repetitions of every stimulation signal amplitude.
Using the common capacitor formula $C=\frac{q}{\Delta v}$, we found that, for the very same neuron, the charge delivered through voltage stimulation is two orders of magnitude larger than the one needed to achieve the same results or stimulation efficiency in current stimulation mode (Figure 4C). Our results show that current stimulation, characterized by a constant rate of charge injection, displays higher efficiency in eliciting neuronal responses with respect to voltage stimulation, which is characterized by an exponential decrease in charge injection (see also Supplementary Figure 1). Results of 3 more neurons confirm the same orders of magnitudes and charge differences for current and voltage stimulation (Supplementary Figure 6). The stimulation efficacy in current and voltage mode is also largely depending on the stimulation buffer implementation (Ballini et al., 2014).

\section{Multi-Electrode Stimulation}

An array of densely distributed electrodes enables to apply different stimulation configurations, either by using the standard single-electrode stimulation approach, or by selecting several 


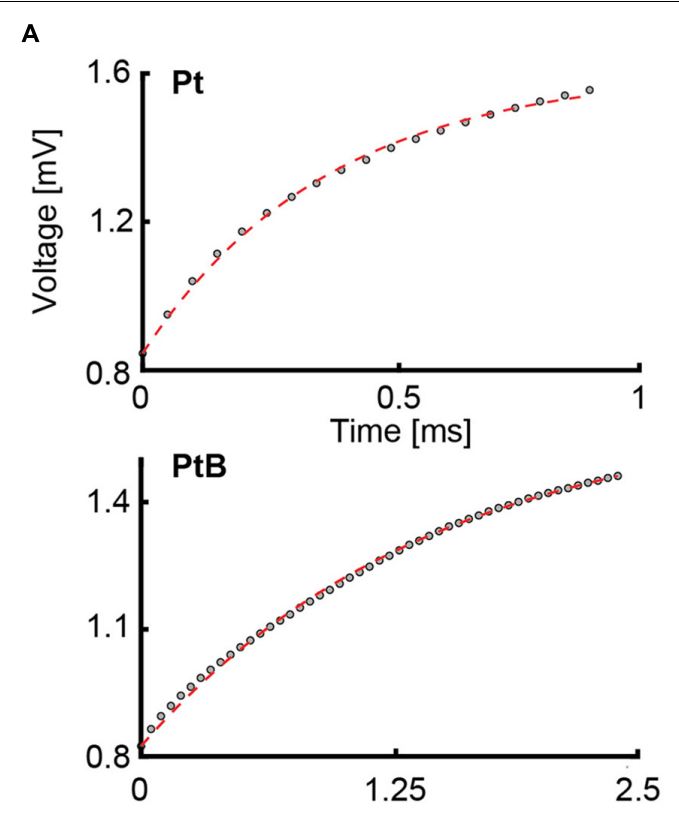

B

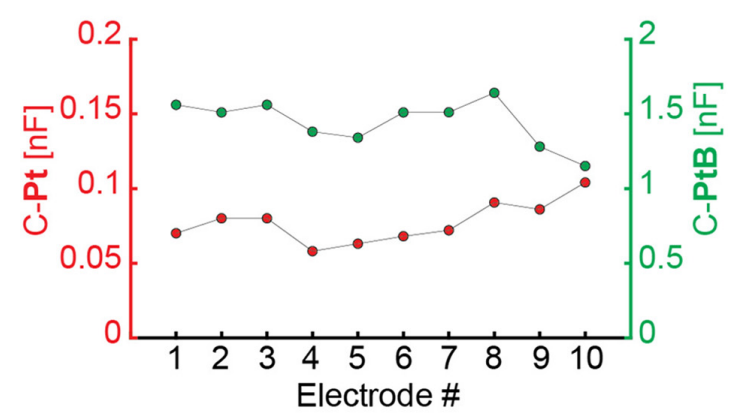

C

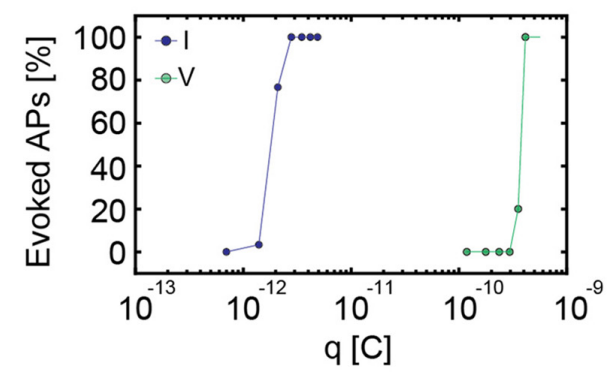

FIGURE 4 | (A) Fitting of an electrode model to the experimental data of measured voltage values upon applying a current stimulus to an electrode. For bright $\mathrm{Pt}(\mathrm{Pt})$, a current stimulation was performed with a biphasic anodic-cathodic waveform with an amplitude of $140 \mathrm{nA}$, a duration of $1 \mathrm{~ms}$ per phase and a readout amplifier gain of 2 . For Pt-black (PtB) the amplitude was $560 \mathrm{nA}$, the duration $2.5 \mathrm{~ms}$ per phase, and the readout amplifier gain was equal to 2 . The different waveforms durations and amplitudes are due to the readout channel saturation in case of bright Pt as a consequence of the higher impedance. In both cases, only the first half of the waveform, i.e., the positive part was used for the fits and is displayed. (B) Capacitance values of $10 \mathrm{Pt}$ and PtB electrodes are presented. The values were computed as a result of the fitting in panel $\mathbf{A}$ by setting the capacitance as an unknown value. (C) Charges required for efficient voltage and current stimulation of the same (Continued)
FIGURE 4 | Continued

neuron. For current stimulation, the waveform had a duration of $20 \mu \mathrm{s}$ per phase, while the duration for voltage stimulation was $100 \mu \mathrm{s}$ per phase. The stimulation protocol included 30 repetitions of every stimulation amplitude in a randomized manner. For current stimulation, the charge was computed as $q=/ \times t$, i.e., the product of applied current and time. For voltage stimulation, the charge was computed as $q=C \times \Delta v$, i.e., the product of the computed capacitance and the voltage change upon stimulation.

electrodes at the same time for applying signals or for grounding. Normally, all unused array electrodes are left floating and do not have a defined potential. The use of neighboring electrodes as stimulation and reference or ground electrodes can produce a locally larger electric field strength, which, in turn, could lead to lower voltages required for stimulation in voltage mode. Moreover, it is possible to stimulate with oppositesign waveforms on adjacent electrodes to reduce and limit stimulation artifacts.

Using COMSOL Multiphysics, we simulated the voltage and electric field distribution on the array for different candidate electrode configurations to see if an increase in electric field strength could increase neuron stimulability and decrease the artifact lateral extension. Three configurations were selected (Figures 5A,B): (i, iv) stimulation with a biphasic voltage waveform $( \pm 100 \mathrm{mV})$ through one electrode against a global reference electrode in solution; (ii, v) stimulation with two neighboring electrodes, using biphasic voltage waveforms $( \pm 100 \mathrm{mV})$ with opposite signs; (iii, vi) stimulation with a biphasic waveform $( \pm 100 \mathrm{mV})$ applied to one electrode, while the neighboring electrode was grounded. All other array electrodes were left floating.

To assess if one of the two chosen configurations with local ground or opposite-sign stimulation signal could improve stimulation efficiency by entailing higher local voltage drops or electric-field strengths, we simulated two probe locations at the right and left side of electrode E1 (Figure 5A), at a height of $1 \mu \mathrm{m}$ above the electrode plane. For configuration (i), one stimulation electrode against a global reference electrode, the voltage drop left and right of the electrode was identical, because the global reference electrode is far away and positioned outside the electrode array. In configuration (iii), the grounded neighboring electrode slightly modified the voltage drop at the right probe location. In configuration (ii), however, the application of a signal to the neighboring second electrode induced a voltage drop decrease at the location of the right probe, as a result of the applied opposite-sign voltages (Figure 5C). However, the electric field between the two electrodes was increased (v) (Figure 5C). Nevertheless, looking at the voltage distribution around the stimulation electrodes suggests comparable results for all configurations, with even a possible decrease in stimulation efficacy for axons that would run through the center region between the two electrodes with opposite-sign waveforms.

To verify the simulation, we then tested the efficacy of these three configurations in evoking APs in neuronal cultures on the HD-MEA. We stimulated six different neurons, after having determined the most reliable electrodes in evoking APs 
A

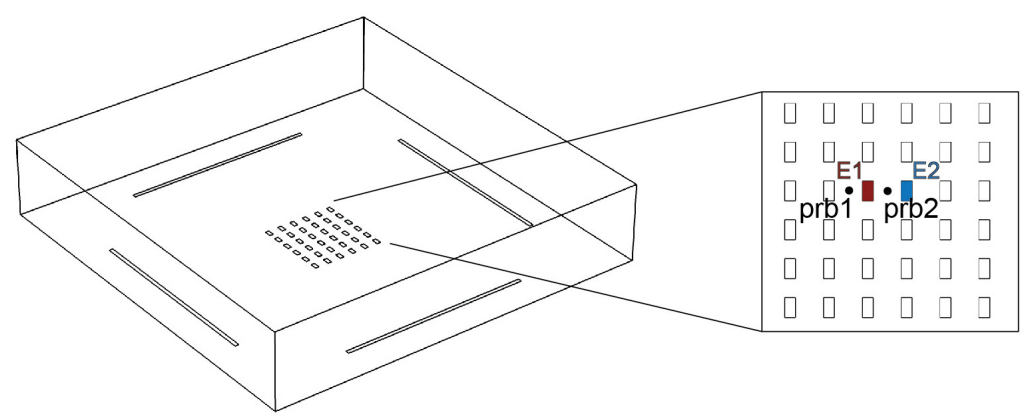

B

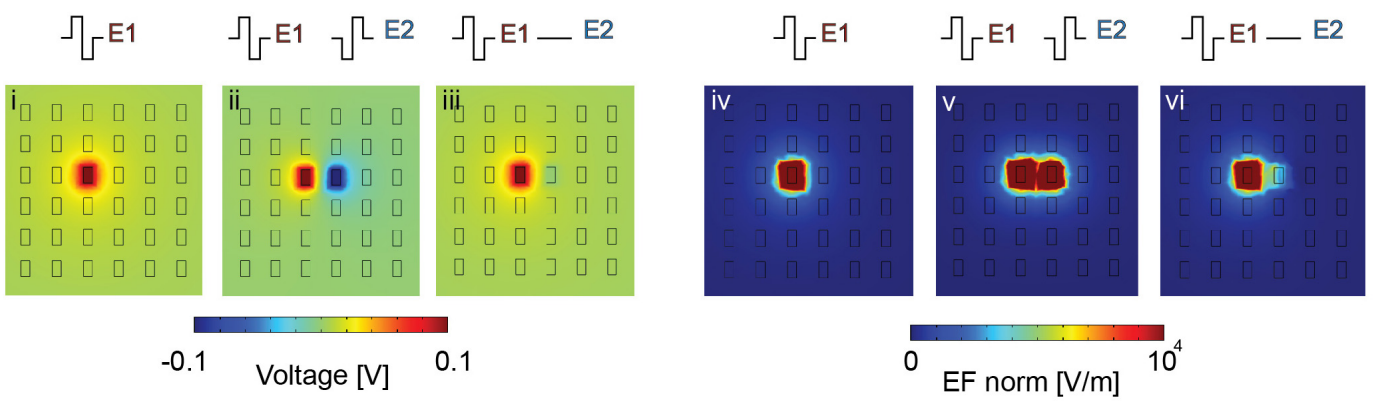

C
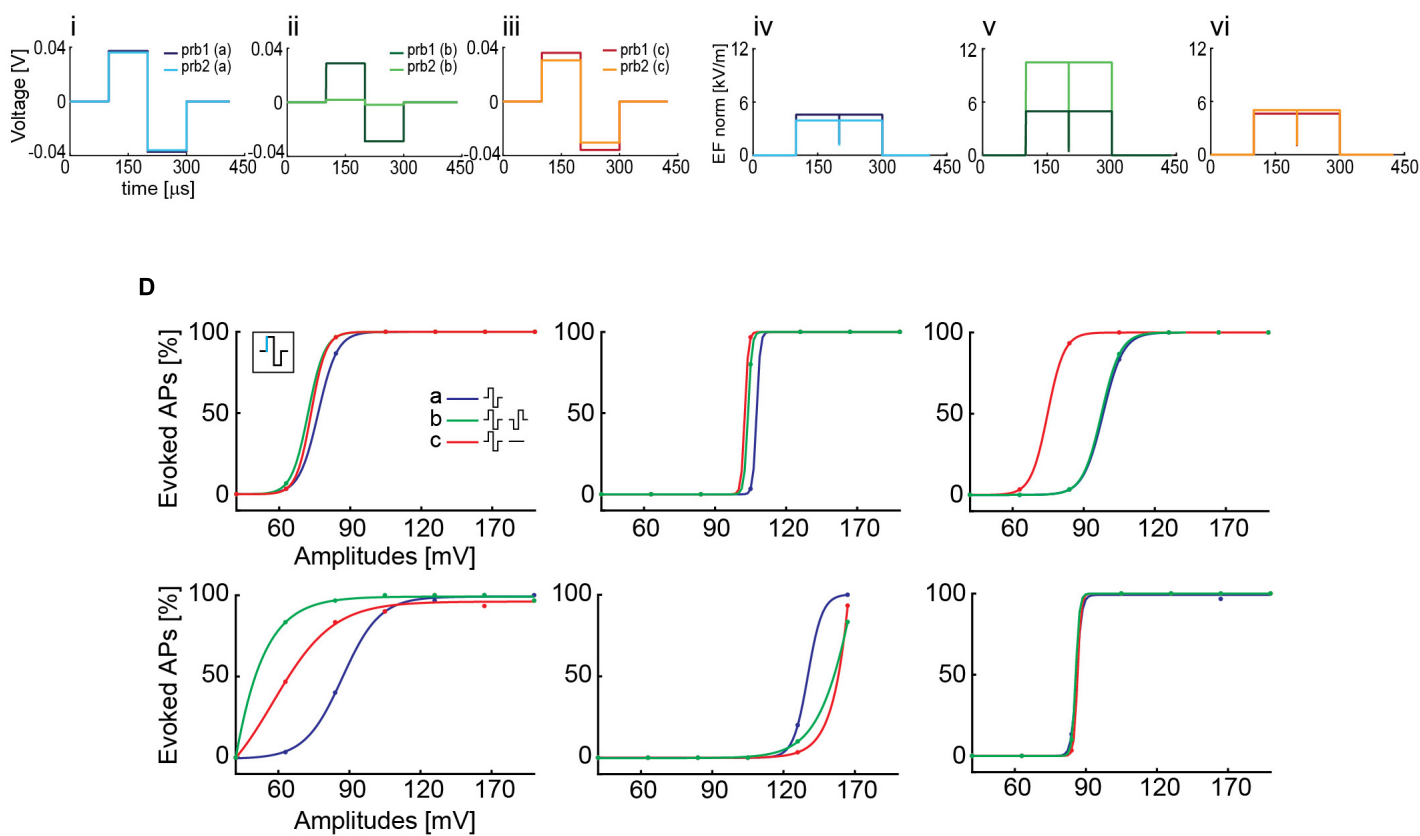

FIGURE 5 | (A) Simplified HD-MEA geometry for COMSOL simulations: 36 planar electrodes at a pitch of $17.5 \mu \mathrm{m}$ and featuring an area of $5 \times 9 \mu \mathrm{m}^{2} ; 4$ reference electrodes were placed at the borders of the array. E1 and E2 denote the electrodes that were used for the simulations in panel $\mathbf{B}$. prb1 and prb2 denote two probe locations, at the right and at the left side of electrode E1, that were used to compute the voltage and the electric-field values for the three configurations in panel $\mathbf{B}$. The two probe locations were chosen so as to compare the effect of using a second array electrode. (B) The simulation was performed in voltage mode, and an amplitude of $\pm 100 \mathrm{mV}$ was used. The voltage and electric field distributions after stimulating with one electrode against a global reference electrode in solution are represented in panels (i) and (iv), those for a stimulation with two neighbored electrodes with synchronized waveforms of opposite signs are represented in panels (ii) and (v), and those for applying a biphasic waveform to one electrode, while the neighboring electrode was grounded are represented in panels (iii) and (vi). The electric field (EF norm) was calculated as $\sqrt{E_{\mathrm{x}}^{2}+E_{\mathrm{y}}^{2}+E_{\mathrm{z}}^{2}}$. (C) Voltage (i, ii, iii) and electric-field (iv, v, vi) distributions at the probe locations in the respective configurations (i-vi) of panel $\mathbf{B}$. The voltage drop, which is responsible for the AP initiation by electrical stimulation, is comparable in all the cases, except for the two-electrode stimulation (ii), where it is somewhat decreased. The electric field is stronger in the case of the two-electrode stimulation (v). (D) Stimulation with a biphasic voltage waveform of six different neurons using the three configurations simulated in COMSOL in panel $\mathbf{B}$. There were differences in the voltage required to evoke activity in the different neurons (range between 60 and $120 \mathrm{mV}$ ), however, there were no major differences for using the three stimulation scenarios explained and displayed in panel $\mathbf{B}$ 
at the respective AISs. First, a randomized voltage stimulation protocol was applied to one electrode, using the global reference electrode at the periphery of the array. A biphasic anodiccathodic voltage stimulation waveform, evidenced to be efficient (Section 3.2), was used with a phase duration of $100 \mu \mathrm{s}$ and an amplitude range between 40 and $160 \mathrm{mV}$. We then repeated the same stimulation protocol by using a grounded reference electrode close to the stimulation electrode to increase the electric field. Finally, we used two electrodes delivering opposite waveforms for stimulation, which further increased the local electric field and, additionally, reduced the lateral extension of the artifact (Supplementary Figure 7). In all the modalities, also the reference electrodes at the sides of the array were left connected. We found differences in the voltage required to evoke activity in the different neurons (range between 60 and $120 \mathrm{mV}$, Figure 5D), however, we did not find major differences for using the three stimulation scenarios explained, simulated and displayed in Figures 5B,C.

This experimental result is in line with the simulations in Figure 5C, which shows comparable extracellular voltage levels for all three configurations. Consequently successful stimulation and APs initiation is not much influenced by applying the three different configurations.

\section{Stimulability Across Cell Development Increases in Early Stages of Neuronal Growth and Development}

To assess if the ability to stimulate neurons is correlated to cell culturing time and AIS growth, we observed and stimulated single neurons during different DIVs. The experimental time points were 14, 17, 20, and $23 \mathrm{DIV}$. We used NeuroFluor NeuO to do live-staining of neurons (Figure 6A). To ensure staining effectiveness, we repeated the staining before every experiment. Isolated cells were identified and three to six stimulation electrodes were used for stimulation. We chose the most efficient stimulation electrode, which was the electrode with the highest extracellular voltage readout (AIS), to execute the stimulation protocol. We used a randomized current stimulation protocol to avoid neuronal adaptation (Grubb and Burrone, 2011). The use of current stimulation was motivated by the reduced artifact (Paragraph 3.1) and the more reliable AP readout (Paragraph 3.2). A biphasic anodic-cathodic waveform was used with a duration of $20 \mu \mathrm{s}$ per phase. We observed that, over 10 DIVs, the neurons moved by a maximum of two electrode distances, which is equal to $35 \mu \mathrm{m}$. In case that the neurons moved by one electrode distance or more, the stimulation electrode was also changed. If the neurons did not move, the most efficient stimulation electrode remained the same (Figure 6A). The movement was evaluated by using the electrical "image" and simultaneous upright confocal imaging. We monitored the soma position with respect to the stimulation electrode position during the experiment days. The results show that the stimulation amplitudes, required to evoke APs, decreased during the first half of the experiment (from DIV 14 to DIV 20), and stabilized around DIV 20 and 23 (Figures 6B,C). An increase in stimulation amplitudes was mostly due to neuron movement and change of the relative position with respect to the stimulation electrode as evident, e.g., in Supplementary Figure 8 for day 20. We also recorded and compared the EAPs of spontaneous neuronal activity over the different DIVs so as to ensure the identity of the respective neurons. To verify if the increase in stimulability was correlated with an AIS growth, we stained neurons at DIV 10, $14,17,20$, and 23 and we computed the length of $N=40$ AIS. We found that there is the tendency of an AIS-average-length increase with increasing DIVs (Figure 6D).

\section{DISCUSSION}

In this study we showed that it is possible to selectively and reliably stimulate individual neurons by applying current and voltage pulses through the 26,400 electrodes of an HDMEA. We tested different stimulation waveforms, durations and amplitudes in voltage and current mode. We demonstrated that we were able to stimulate individual neurons by combining high-density recording of single-neuron action potentials with immunostaining and confocal microscopy. Previous studies (Wagenaar et al., 2004) reported that, in voltage mode, the biphasic anodic-cathodic waveform was most efficient for selective stimulation, followed by the cathodic-anodic one. We found that the most efficient stimulation waveform is the monophasic cathodic waveform, followed by the biphasic anodiccathodic, by comparing the $\mathrm{V}_{\mathrm{pp}}$ for the same pulse width (Figure 2). It is, however, important to note that we used significantly lower stimulation voltages $\left(80 \mathrm{mV}_{\mathrm{pp}}\right)$, as we could target the most sensitive region of a neuron, the AIS, for stimulation. Moreover, we used much smaller electrodes with respect to the $30 \mu \mathrm{m}$ diameter electrodes in the study by Wagenaar et al. (2004). In current mode, we confirmed that the triphasic waveform is an efficient stimulation signal that produces a comparably smaller artifacts than the biphasic one (Grosberg et al., 2017). In comparison to previous findings, we were able to improve selectivity due to the high electrode density and the inherent possibility of directed targeting of neurons and their AISs (Radivojevic et al., 2016). We, hence, could stimulate with comparably low charges of a few picoCoulombs (Figure 3). Moreover, the small electrodes provided sufficient stimulation charge density also for small applied voltages.

In comparing current and voltage stimulation parameters for our HD-MEA, we found that a waveform duration of $100 \mu$ s per phase was required to efficiently evoke EAPs in voltage mode. In current mode, instead, a duration of only $20 \mu \mathrm{s}$ per phase was required, which entailed a shorter artifact duration. However, it needs to be mentioned that the possible shorter signal duration in current mode was also a consequence of the settling time of our stimulation buffers. According to our measurements, current stimulation is the preferable stimulation modality, which is in line with reports in literature (Grosberg et al., 2017; Fan et al., 2019). By using current stimulation it was possible to use already the next neighboring electrodes, at $17.5 \mu \mathrm{m}$ pitch from the stimulation electrode, for EAP recording (Figures 1, 2). Owing to the high spatial resolution and dense electrode packing of the array, it was possible to read out electrical activity at the cell soma 
A

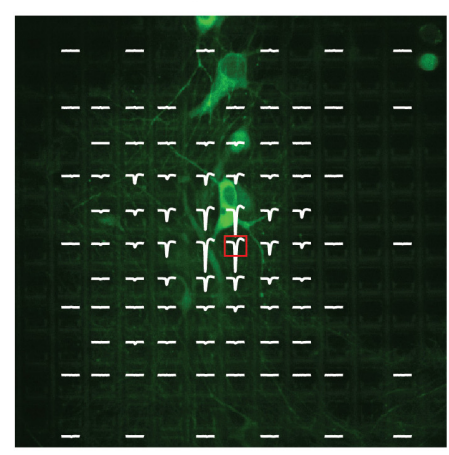

B

D
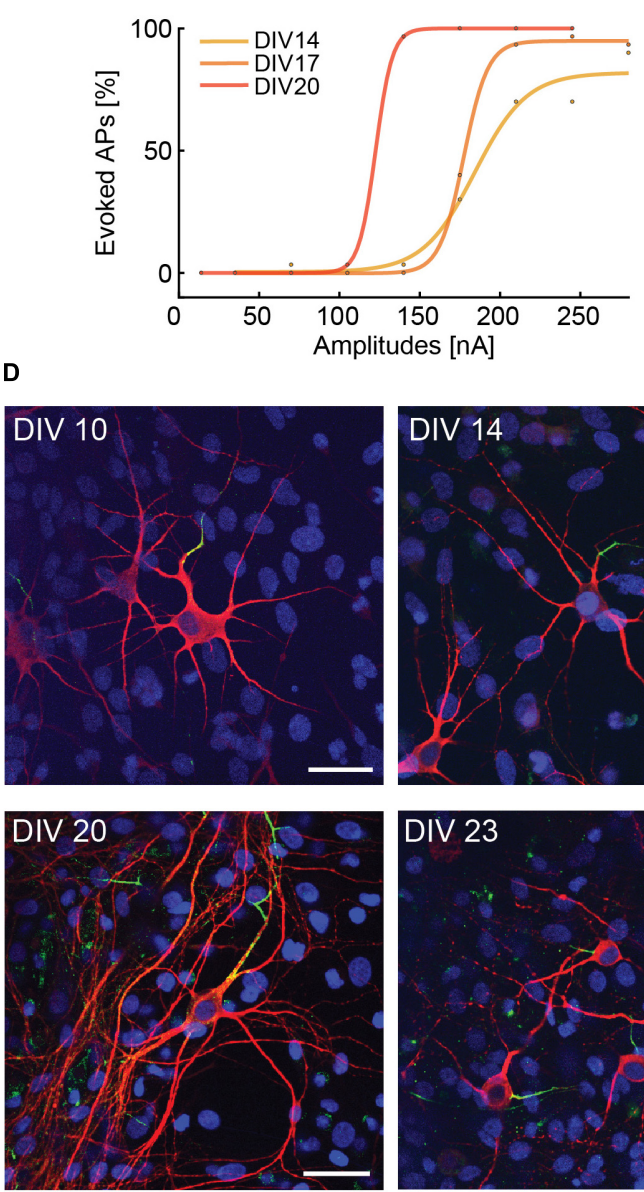

DIV 17

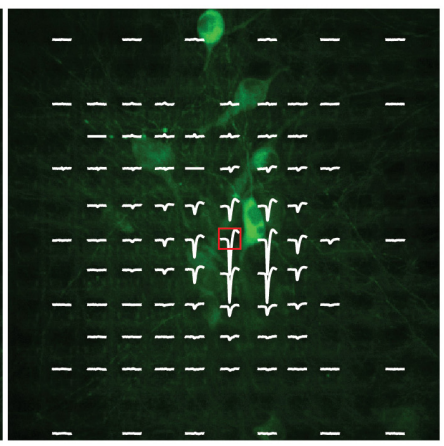

DIV 20

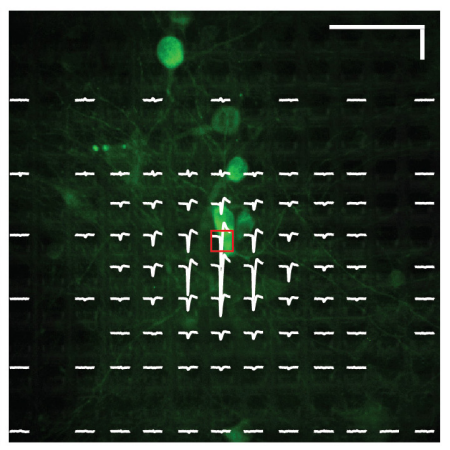

C

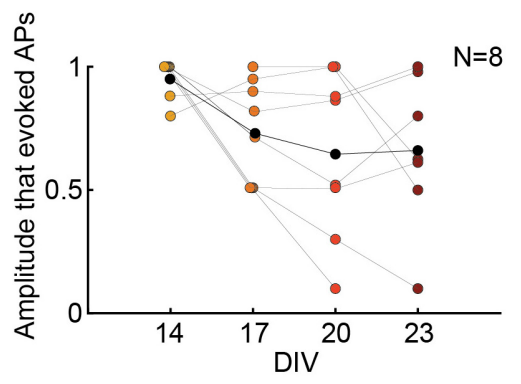

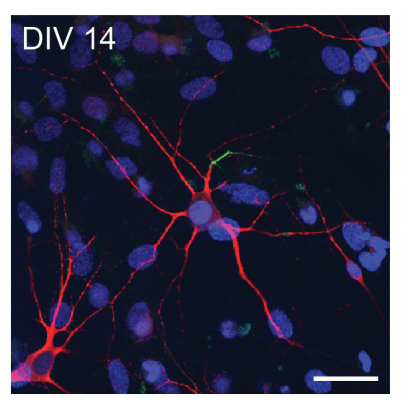
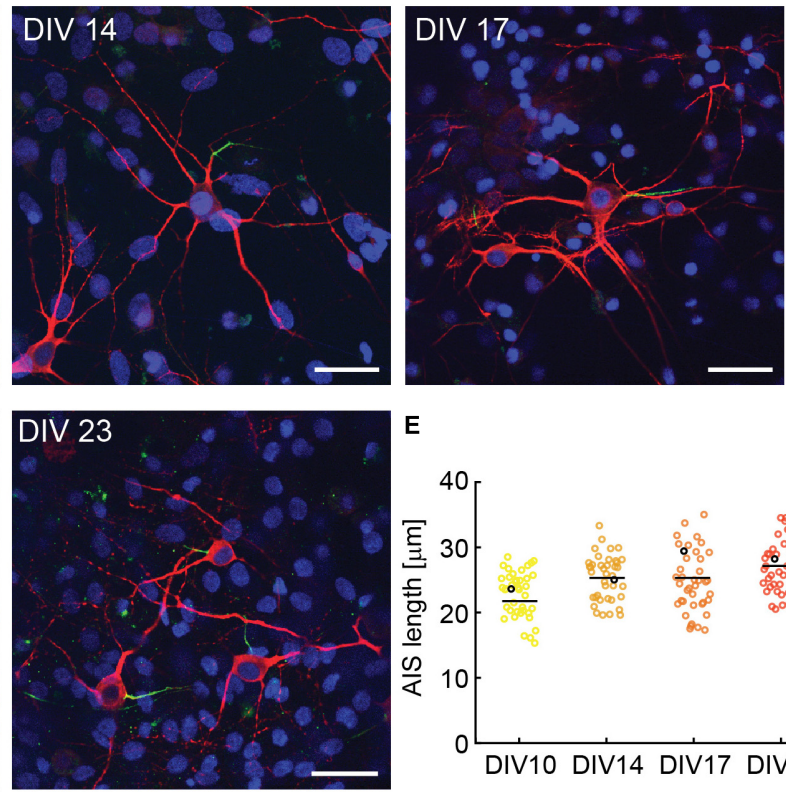

E

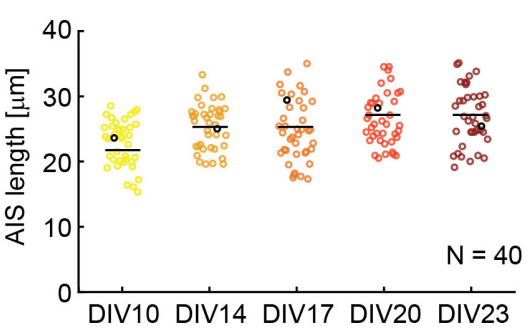

FIGURE 6 | (A) Superimposed fluorescence image and electrical footprint of the same neuron at DIVs 14, 17, 20. The stimulation electrodes used for the analysis are indicated with a red box. The stimulation electrode changed position with the neuron movements during the experiment. The signals recorded on the different electrodes are displayed in white (electrical footprint). Horizontal scale bar: $50 \mu \mathrm{m}$, vertical scale bar: $100 \mu \mathrm{V}$. (B) Current stimulation activation curves of the neuron in panel $\mathbf{A}$ over time (DIVs). The stimulation amplitudes necessary to evoke APs decreased between DIV 14 and 20 . (C) Stimulability experiments for $N=8$ neurons. For every neuron, the stimulation amplitudes were normalized to the highest amplitude that was necessary to evoke APs $90 \%$ of times over 30 repetitions during the DIVs 14-23. The stimulability varied from neuron to neuron. We observed a trend of decreased stimulation amplitudes between DIV 14 and 20 that then stabilization between DIV 20 and 23. The average relative stimulation current amplitude is represented with black dots. (D) Sample microscopy stainings showing fixed neurons at DIV 10, 14, 17, 20, 23. The nuclei are represented in blue, the neurons in red and the AISs in green. The 5 neurons are represented as black dots in panel E. Scale bar $35 \mu \mathrm{m}$. (E) AIS lengths and length distribution over time: DIVs 10, 14, 17, 20, 23. The average length tends to slightly increase over time in vitro ( $N=40)$. 
while stimulating the AIS of the very same cell, so that it was possible to accurately estimate stimulation success and efficiency.

Based on our experiments, we estimated current stimulation to be more efficient than voltage stimulation in evoking APs using our HD-MEA. To further test this assumption, we determined the electrode impedances by measuring and modeling the voltage readout upon applying current stimulation (Franks et al., 2005). The fit of the measurement data to the model returned capacitance values of $\sim 1.4 \mathrm{nF}$ for Pt-black and $\sim 0.07 \mathrm{nF}$ for bright $\mathrm{Pt}$ electrodes. We used these capacitance values to compare voltage and current activation curves, and found that the charge needed to evoke APs in current mode is, indeed, by two orders of magnitude lower than in voltage mode (Figure 4). The stimulation efficacy in current and voltage mode is also depending on the stimulation buffer implementation (Ballini et al., 2014).

We then compared different electrode configurations, which provided increased electric field strengths, as compared to using a single electrode against a global reference electrode in solution. As shown in Figure 5D, the stimulation efficacy, however, was found to be in the same range for different neurons.

Capitalizing on the short artifact duration upon using current mode, we finally studied neuron stimulability during cell development and growth. Our hypothesis was that the AIS development over time would increase neuron stimulability. We combined live stainings and electrical recordings/stimulation to follow neuronal development over several days. The most challenging procedure during the experiments was to identify more or less isolated neurons on the array and in the culture and to then track them over several days during the experiments. Several neurons had to be discarded as a consequence of cell death during the experiments or because we could not track them over the experiment time. Nevertheless, we observed a decrease in stimulation amplitudes to evoke APs, which was correlated to AIS growth in length (Figure 6).

In summary, this work presents a comprehensive study on electrical stimulation with microelectrodes of HD-MEAs and shows ways to realize single-neuron stimulation. Selecting optimal stimulation parameters could prove to be powerful for other in vitro applications, such as the control of neuralnetwork bursting through electrical stimulation (Wagenaar et al., 2005), or for ex vivo stimulations, for example, in retinal preparations or brain slices. We think that in vivo stimulation methods (epiretinal implants) could also benefit from findings of this paper in order to implement targeted stimulation of individual neurons. The delivered charges to depolarize neuronal membranes amounted to $0.02 \mathrm{pC} / \mu \mathrm{m}^{2}$ with our HD-MEA, while,

\section{REFERENCES}

Ahmadian, Y., Packer, A. M., Yuste, R., and Paninski, L. (2011). Designing optimal stimuli to control neuronal spike timing. J. Neurophysiol. 106, 1038-1053. doi: 10.1152/jn.00427.2010

Ahuja, A. K., Yeoh, J., Dorn, J. D., Caspi, A., Wuyyuru, V., McMahon, M. J., et al. (2013). Factors affecting perceptual threshold in Argus II retinal prosthesis subjects. Transl. Vis. Sci. Technol. 2:1. doi: 10.1167 tvst.2.4.1 for example, retinal implants currently work with $3.5 \mathrm{pC} / \mu \mathrm{m}^{2}$ (Ahuja et al., 2013). A small size of electrodes and their dense packing may prove beneficial to stimulate neurons and could improve stimulation accuracy of prosthetic implants while enabling lower power consumption.

\section{DATA AVAILABILITY}

The datasets generated for this study are available on request to the corresponding author.

\section{AUTHOR CONTRIBUTIONS}

SR, MF, JM, VV, UF, and AH performed experimental design. SR and CM performed experiments and data analysis. MF, JM, and VV provided technical support. SR and $\mathrm{AH}$ wrote the manuscript. MF, CM, VV, JM, and UF reviewed the manuscript. $\mathrm{AH}$ supervised the project.

\section{FUNDING}

This work was supported by the European Community through the European Research Council Advanced Grant 694829 "neuroXscales" and the Swiss National Science Foundation Grant 205321_157092/1 (“Axons"). The funders had no role in study design, data collection and analysis, decision to publish, or preparation of the manuscript.

\section{ACKNOWLEDGMENTS}

We thank Alexander Stettler and Peter Rimpf for post-processing CMOS chips. We also thank the D-BSSE support staff for help with the experiments, in particular Mario Modena and Massimiliano Gusmaroli for COMSOL support and Xinyue Yuan for electronics simulations. Finally, we are indebted to Prof. Edward Zellers, University of Michigan, for valuable comments on the manuscript.

\section{SUPPLEMENTARY MATERIAL}

The Supplementary Material for this article can be found online at: https://www.frontiersin.org/articles/10.3389/fnins. 2019.00208/full\#supplementary-material

Armenta Salas, M., Bashford, L., Kellis, S., Jafari, M., Jo, H., Kramer, D., et al. (2018). Proprioceptive and cutaneous sensations in humans elicited by intracortical microstimulation. eLife 7:e32904. doi: 10.7554/eLife. 32904

Bakkum, D. J., Obien, M. E. J., Radivojevic, M., Jäckel, D., Frey, U., Takahashi, H., et al. (2018). The axon initial segment is the dominant contributor to the neuron's extracellular electrical potential landscape. Adv. Biosyst. 3:1800308. doi: 10.1002/adbi.2018 00308 
Ballini, M., Muller, J., Livi, P., Chen, Y., Frey, U., Stettler, A., et al. (2014). A 1024channel CMOS microelectrode array with 26,400 electrodes for recording and stimulation of electrogenic cells in vitro. IEEE J. Solid State Circuits 49, 2705-2719. doi: 10.1109/JSSC.2014.2359219

Benabid, A. L., Chabardes, S., Mitrofanis, J., and Pollak, P. (2009). Deep brain stimulation of the subthalamic nucleus for the treatment of Parkinson's disease. Lancet Neurol. 8, 67-81. doi: 10.1016/S1474-4422(08) 70291-6

Berdondini, L., Imfeld, K., MacCione, A., Tedesco, M., Neukom, S., KoudelkaHep, M., et al. (2009). Active pixel sensor array for high spatiotemporal resolution electrophysiological recordings from single cell to large scale neuronal networks. Lab Chip 9, 2644-2651. doi: 10.1039/b907 $394 \mathrm{a}$

Bertotti, G., Velychko, D., Dodel, N., Keil, S., Wolansky, D., Tillak, B., et al. (2014). "A CMOS-based sensor array for in-vitro neural tissue interfacing with 4225 recording sites and 1024 stimulation sites," in Proceedings of the IEEE Biomedical Circuits and Systems Conference (BioCAS), Lausanne, 304-307. doi: 10.1109/ BioCAS.2014.6981723

Brindley, G. S., and Lewin, W. S. (1968). The sensations produced by electrical stimulation of the visual cortex. J. Physiol. 196, 479-493. doi: 10.1113/jphysiol. 1968.sp008519

Dagnelie, G. (2012). Retinal implants: emergence of a multidisciplinary field. Curr. Opin. Neurol. 25, 67-75. doi: 10.1097/WCO.0b013e3283 $4 \mathrm{f0} 2 \mathrm{c} 3$

Eversmann, B., Jenkner, M., Hofmann, F., Paulus, C., Brederlow, R., Holzapfl, B., et al. (2003). A $128 \times 128$ CMOS bio-sensor array for extracellular recording of neural activity. IEEE J. Solid State Circuits 38, 2306-2317. doi: 10.1109/JSSC. 2003.819174

Fan, V. H., Grosberg, L. E., Madugula, S. S., Hottowy, P., Dabrowski, W., Sher, A., et al. (2019). Epiretinal stimulation with local returns enhances selectivity at cellular resolution. J. Neural Eng. doi: 10.1088/1741-2552/aaeef1 [Epub ahead of print].

Franks, W., Schenker, I., Schmutz, P., and Hierlemann, A. (2005). Impedance characterization and modeling of electrodes for biomedical applications. IEEE Trans. Biomed. Eng. 52, 1295-1302. doi: 10.1109/TBME.2005. 847523

Frey, U., Sedivy, J., Heer, F., Pedron, R., Ballini, M., Mueller, J., et al. (2010). Switch-matrix-based high-density microelectrode array in CMOS technology. IEEE J. Solid State Circuits 45, 467-482. doi: 10.1109/JSSC.2009. 2035196

Greenberg, R. J., De Juan, E., Humayun, M. S., McClure, K. H., Talbot, N. H., Neysmith, J. M., et al. (2018). Aggregate electrode for neural stimulation. U.S. Patent No 20,180,104,487. Sylmar, CA: Second Sight Medical Products.

Grosberg, L. E., Ganesan, K., Goetz, G. A., Madugula, S. S., Bhaskhar, N., Fan, V., et al. (2017). Activation of ganglion cells and axon bundles using epiretinal electrical stimulation. J. Neurophysiol. 118, 1457-1471. doi: 10.1152/jn.00750. 2016

Grubb, M. S., and Burrone, J. (2011). Activity-dependent relocation of the axon initial segment fine- tunes neuronal excitability. Nature 465, 1070-1074. doi: 10.1038/nature09160.Activity-dependent

Héduit, A., Quinio, I., Stadmuller, D., and Thévenot, D. R. (1996). Modified platinum electrodes: electrochemical characteristics and behaviour in activated sludge. Water Sci. Technol. 34, 143-150. doi: 10.2166/wst.1996. 0427

Hill, D. N., Mehta, S. B., and Kleinfeld, D. (2011). Quality metrics to accompany spike sorting of extracellular signals. J. Neurosci. 31, 8699-8705. doi: 10.1523/ JNEUROSCI.0971-11.2011

Hottowy, P., Skoczeń, A., Gunning, D. E., Kachiguine, S., Mathieson, K., Sher, A., et al. (2012). Properties and application of a multichannel integrated circuit for low-artifact, patterned electrical stimulation of neural tissue. J. Neural Eng. 9:066005. doi: 10.1088/1741-2560/9/6/ 066005

Joye, N., Schmid, A., and Leblebici, Y. (2009). Electrical modeling of the cell-electrode interface for recording neural activity from high-density microelectrode arrays. Neurocomputing 73, 250-259. doi: 10.1016/j.neucom. 2009.09.006
Kumar, S. S., Wülfing, J., Okujeni, S., Boedecker, J., Riedmiller, M., and Egert, U. (2016). Autonomous optimization of targeted stimulation of neuronal networks. PLoS Comput. Biol. 12:e1005054. doi: 10.1371/journal.pcbi. 1005054

Merrill, D. R. (2010). "The electrochemistry of charge injection at the electrode/tissue interface," in Implantable Neural Prostheses 2: Techniques and Engineering Approaches, eds D. Zhou and E. Greenbaum (New York, NY: Springer), 85-138. doi: 10.1007/978-0-38798120-8_4

Obien, M. E. J., Deligkaris, K., Bullmann, T., Bakkum, D. J., and Frey, U. (2015). Revealing neuronal function through microelectrode array recordings. Front. Neurosci. 9:423. doi: 10.3389/fnins.2014. 00423

Oldham, K. B. (2008). A Gouy-Chapman-Stern model of the double layer at a (metal)/(ionic liquid) interface. J. Electroanal. Chem. 613, 131-138. doi: 10. 1016/j.jelechem.2007.10.017

Perlmutter, J. S., and Mink, J. W. (2006). Deep brain stimulation. Annu. Rev. Neurosci. 29, 229-257. doi: 10.1146/annurev.neuro.29.051605. 112824

Radivojevic, M., Jäckel, D., Altermatt, M., Müller, J., Viswam, V., Hierlemann, A., et al. (2016). Electrical identification and selective microstimulation of neuronal compartments based on features of extracellular action potentials. Sci. Rep. 6:31332. doi: 10.1038/srep 31332

Raspopovic, S., Capogrosso, M., Petrini, F. M., Bonizzato, M., Rigosa, J., Di Pino, G., et al. (2014). Restoring natural sensory feedback in real-time bidirectional hand prostheses. Sci. Transl. Med. 6:222ra19. doi: 10.1126/ scitranslmed.3006820

Rattay, F. (1999). The basic mechanism for the electrical stimulation of the nervous system. Neuroscience 89, 335-346. doi: 10.1016/S0306-4522(98) 00330-3

Sekirnjak, C., Hottowy, P., Sher, A., Dabrowski, W., Litke, A. M., and Chichilnisky, E. J. (2008). High-resolution electrical stimulation of primate retina for epiretinal implant design. J. Neurosci. 28, 4446-4456. doi: 10.1523/ JNEUROSCI.5138-07.2008

Shannon, R. V. (1983). Multichannel electrical stimulation of the auditory nerve in man. I. Basic psychophysics. Hear. Res. 11, 157-189.

Shannon, R. V. (1985). Threshold and loudness functions for pulsatile stimulation of cochlear implants. Hear. Res. 18, 135-143. doi: 10.1016/0378-5955(85) 90005-X

Sharma, P., and Bhatti, T. S. (2010). A review on electrochemical double-layer capacitors. Energy Convers. Manag. 51, 2901-2912. doi: 10.1016/j.enconman. 2010.06.031

Thomas, C. A., Loeb, G. E., and Okunj, L. M. (1972). A miniature microelectrode array to monitor the bioelectric activity of cultured cells. Exp. Cell Res. 74, 61-66. doi: 10.1016/0014-4827(72) 90481-8

Tsai, D., Chen, S., Protti, D. A., Morley, J. W., Suaning, G. J., and Lovell, N. H. (2012). Responses of retinal ganglion cells to extracellular electrical stimulation, from single cell to population: model-based analysis. PLoS One 7:e53357. doi: 10.1371/journal.pone. 0053357

Tsai, D., Sawyer, D., Bradd, A., Yuste, R., and Shepard, K. L. (2017). Addendum: a very large-scale microelectrode array for cellular-resolution electrophysiology. Nat. Commun. 8:4497. doi: 10.1038/s41467-01702009-x

Viswam, V., Dragas, J., Shadmani, A., Chen, Y., Stettler, A., Muller, J., et al. (2016). "Multi-functional microelectrode array system featuring 59,760 electrodes, 2048 electrophysiology channels, impedance and neurotransmitter measurement units," in Proceedings of the IEEE International Solid-State Circuits Conference (ISSCC), Vol. 59, San Francisco, CA, 394-396. doi: 10.1109/ISSCC.2016. 7418073

Wagenaar, D. A., Madhavan, R., Pine, J., and Potter, S. M. (2005). Controlling bursting in cortical cultures with closed-loop multi-electrode stimulation. J. Neurosci. 25, 680-688. doi: 10.1523/JNEUROSCI.4209-04. 2005 
Wagenaar, D. A., Pine, J., and Potter, S. M. (2004). Effective parameters for stimulation of dissociated cultures using multi-electrode arrays. J. Neurosci. Methods 138, 27-37. doi: 10.1016/j.jneumeth.2004.03.005

Weiland, J. D., Anderson, D. J., and Humayun, M. S. (2002). In vitro electrical properties for iridium oxide versus titanium nitride stimulating electrodes. IEEE Trans. Biomed. Eng. 49, 1574-1579. doi: 10.1109/TBME.2002. 805487

Woodson, E. A., Reiss, L. A. J., Turner, C. W., Gfeller, K., and Gantz, B. J. (2009). The hybrid cochlear implant: a review. Adv. Otorhinolaryngol. 67, 125-134. doi: $10.1159 / 000262604$

Wülfing, J., Kumar, S. S., Boedecker, J., Riedmiller, M., and Egert, U. (2018), "Controlling biological neural networks with deep reinforcement learning," in Proceedings of the European Symposium on Artificial Neural Networks, Computational Intelligence and Machine Learning, Bruges, 25-27.
Conflict of Interest Statement: MF, JM, and UF are co-founders of MaxWell Biosystems AG, which commercializes HD-MEA technology.

The remaining authors declare that the research was conducted in the absence of any commercial or financial relationships that could be construed as a potential conflict of interest.

Copyright $\odot 2019$ Ronchi, Fiscella, Marchetti, Viswam, Müller, Frey and Hierlemann. This is an open-access article distributed under the terms of the Creative Commons Attribution License (CC BY). The use, distribution or reproduction in other forums is permitted, provided the original author(s) and the copyright owner(s) are credited and that the original publication in this journal is cited, in accordance with accepted academic practice. No use, distribution or reproduction is permitted which does not comply with these terms. 